\title{
Person Retrieval in Surveillance Using Textual Query: A Review
}

\author{
Hiren Galiyawala • Mehul S. Raval
}

Received: date / Accepted: date

\begin{abstract}
Recent advancement of research in biometrics, computer vision, and natural language processing has discovered opportunities for person retrieval from surveillance videos using textual query. The prime objective of a surveillance system is to locate a person using a description, e.g., a short woman with a pink t-shirt and white skirt carrying a black purse. She has brown hair. Such a description contains attributes like gender, height, type of clothing, colour of clothing, hair colour, and accessories. Such attributes are formally known as soft biometrics. They help bridge the semantic gap between a human description and a machine as a textual query contains the person's soft biometric attributes. It is also not feasible to manually search through huge volumes of surveillance footage to retrieve a specific person. Hence, automatic person retrieval using vision and language-based algorithms is becoming popular. In comparison to other state-of-the-art reviews, the contribution of the paper is as follows: 1. Recommends most discriminative soft biometrics for specific challenging conditions. 2. Integrates benchmark datasets and retrieval methods for objective performance evaluation. 3. A complete snapshot of techniques based on features, classifiers, number of soft biometric attributes, type of the deep neural networks, and performance measures. 4. The comprehensive coverage of person retrieval from handcrafted features based methods to end-to-end approaches based on natural language description.
\end{abstract}

Keywords Attribute recognition · natural language description · person retrieval · soft biometric attributes · video surveillance.

Hiren Galiyawala

School of Engineering and Applied Science,

Ahmedabad University, India.

E-mail: hirenkumar.g@ahduni.edu.in

Mehul S. Raval

School of Engineering and Applied Science,

Ahmedabad University, India.

E-mail: mehul.raval@ahduni.edu.in 


\section{Introduction and motivation}

Security of the individual and society is a significant concern in today's world of automation. Modern life of human beings involves many Internet-of-Things (IoT) based devices to interact and operate. While such devices provide luxury to live, they may also endanger living by compromising privacy. There are many questions in daily life such as:

- Is this person authorized to enter into specific premises?

- Is this person authorized to access the mobile application?

- Is the right person accessing the system?

- Is the person a legal resident of the country?

Answers to such questions need monitoring using surveillance networks. Most countries in the world have surveillance cameras in many places. Camera networks cover many places like housing colonies, small offices, large corporate offices, retail shops, supermarkets, public places like airports, railway stations, and sports venues. The world has observed many events like terrorist attacks and general strikes in recent years. Public safety is the most critical task to be carried out by a security agency during such an event. A surveillance network helps investigators during and after the event. Person localisation plays a significant role during such an investigation and helps to accelerate the process of investigation. Typically, video operators carry out the person(s) retrieval task by searching through a video database. It takes time, and the process is also very inefficient. Thus, intelligent video surveillance is growing as an active research area.

Some critical definitions for giving the reader a better understanding are as follows:

Person identification: It is a process to establish an individual's identity or to identify an individual uniquely. Biometrics such as fingerprint and face are unique to an individual, which helps in person identification.

Person re-identification (Re-ID): Re-ID aims to identify a person in a new place and at a different time captured by another camera. The query can be in the form of an image, video, and even text description. It is useful to re-identify a person in surveillance-based applications.

Person retrieval aims to spot the person of interest from an image gallery or video frame given the query. It does not seek to establish an identity; instead, it reduces the search space. Thus, the output may contain multiple retrievals matching the query. For example, a surveillance frame may have multiple persons with a description, i.e., a man with a blue t-shirt and black jeans. 


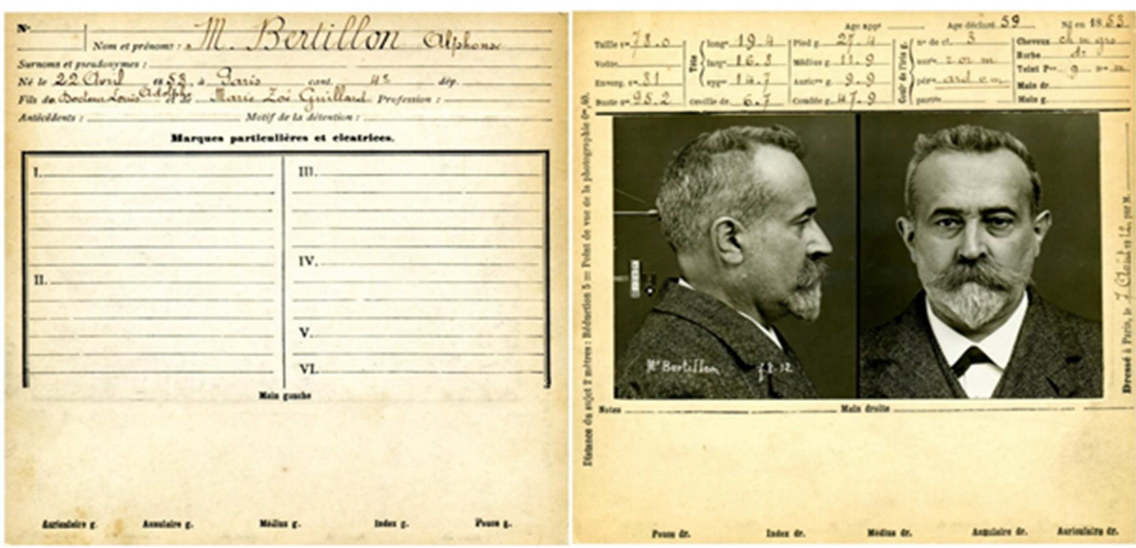

Fig. 1 Anthropometric data sheet of Alphonse Bertillon [3].

\subsection{History and background}

An early person identification system developed by Alphonse Bertillon, a police officer and biometric researcher from Paris, France, identified criminals in the 19th century. His approach is also known as Bertillonage [1], which was widely accepted in France and other countries. Criminal identification was made only by employing photographs or names before Bertillonage. He was the first to use anthropometry for law enforcement using various physical measurements. He also defined the process of physical measurements and photograph acquisition to maintain uniformity in the records $[1,2]$ (see Fig. 1). Bertillon's system measures physical features like standing height, length and breadth of head, length of individual fingers, dimensions of the foot, dimensions of the nose, and ear. It also has descriptions of eye and hair colour, any scars, marks, and tattoos on the body [1].

Two persons may likely have the same height, but the chances of other measurements being similar is highly unlikely. Sizes are not unique to individuals and hence may fail to identify a person if used without other attributes. e.g., twins may have similar biological features. Hence, it has often been superseded by fingerprint-based identification. Biometrics is a reliable solution for person identification [4] due to properties like uniqueness, stability, universality, and accessibility. The answer is dependent on "what you are" which uses face, fingerprint, palm print, hand geometry. It is independent of "what you possess" like identity card, or "what you know" e.g., password or the personal identification number $[15,16]$. Such traditional biometrics-based systems are successful in many applications like forensics, employee attendance, and mobile or laptop security. Biometrics-based retrieval systems have limited usage in surveillance applications due to the following:

- Biometric samples are difficult to capture from video footage of unconstrained environments. 


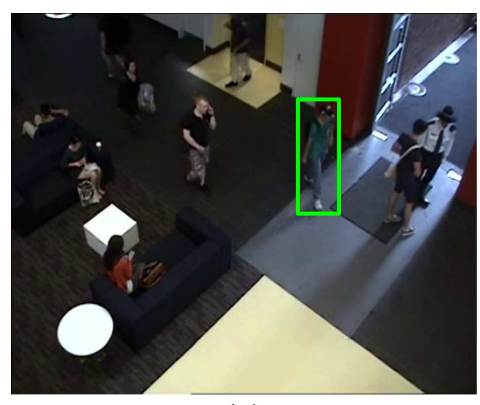

(a)

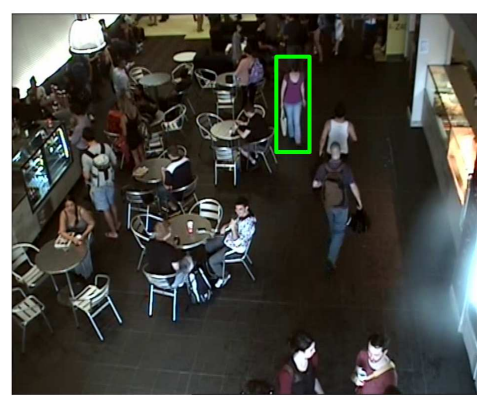

(b)

Fig. 2 Video surveillance frame samples [5].

- It is challenging to capture physiological samples (e.g., fingerprint) from an uncooperative person.

- Biometric attributes (e.g., face) are challenging to acquire due to camera distance and position.

- Captured biometrics have poor quality due to low-resolution cameras.

Traditional biometric-based systems fail to retrieve a person from surveillance videos due to the above reasons. Fig. 2 shows sample video surveillance frames from the AVSS 2018 challenge II database [5]. A person of interest is within a green bounding box. It shows various scenarios where traditional biometrics-based systems fail to retrieve the person. Fig. 2(a) shows the environment with poor illumination and a low-resolution frame. Fig. 2(b) shows a scenario with a crowd and a large distance between the camera and the person. Face recognition-based systems fail to retrieve the person in such scenarios. However, attributes like colour and type of clothing, height and gender can help in-person retrieval under such challenging conditions. For example, green (in Fig. 2(a)) and purple (in Fig. 2(b)) coloured clothes are identifiable. Jain et al. [6] introduce such personal attributes as soft biometrics, and many other research articles elaborate on soft biometrics [6-13]. Boston Marathon bombing [14] is a real-world example where police investigation took three days to search out the suspect from hundreds of hours of surveillance videos. Thus, automation of the person retrieval process saves time during a critical investigation. With the ever-increasing demand for security through surveillance, researchers are infusing more interest in developing soft biometric-based person retrieval from low-quality surveillance videos where traditional biometrics fail.

Biometrics are broadly classified as hard and soft biometrics, as shown in Fig. 3. Hard biometrics have physiological and behavioural attributes of a person. Physiologically based methods establish a person's identity using physiological characteristics like face, iris, fingerprint, palm print, hand geometry and DNA. Behavioural techniques perform authentication by recognizing patterns such as voice, gait, and signature. Although biometrics like face, fingerprint, and palm print are unique to an individual, a single biometric cannot 


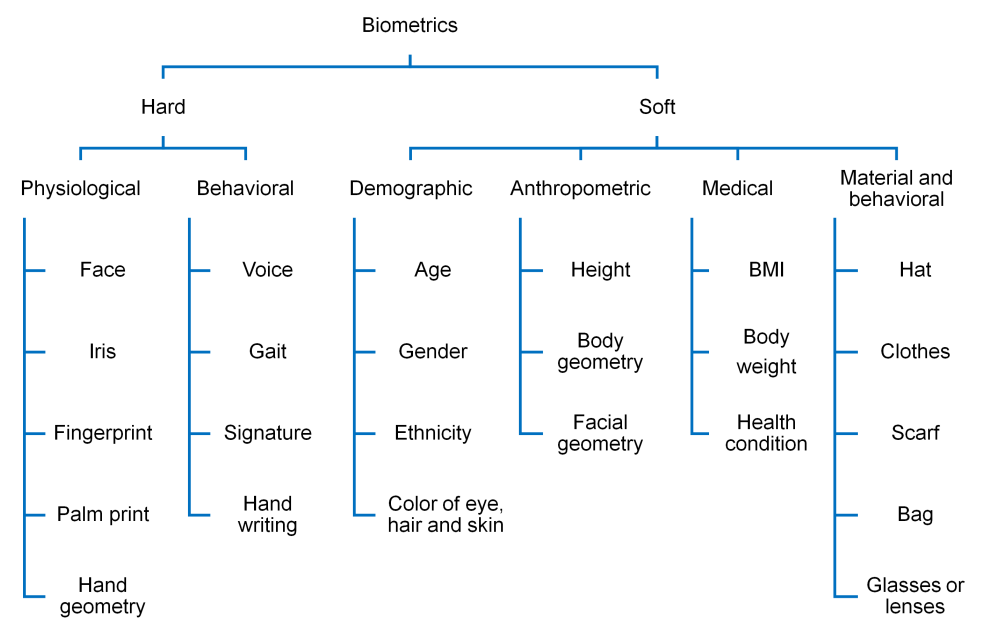

Fig. 3 Type of biometrics.

meet the requirements of all applications $[15,16]$. For example, camera-based security systems cannot use fingerprints as a biometric for user authentication. Thus, biometric attribute selection depends on the application. One such application is person retrieval from a distance through a surveillance frame. Here traditional biometrics fail, but soft biometrics play a critical role in retrieval. Soft biometrics are the ancillary information deduced from the human body. Dantcheva et al. [12] categorized soft biometrics into demographic, anthropometric, medical, material, and behavioural attributes.

The term demographic is related to the accumulation and statistical analysis of broad characteristics about groups of people and populations. Age, ethnicity, gender, and race are such characteristics that are used as soft biometrics and also termed as global traits [17]. Gender, ethnicity and race usually do not show changes over the person's lifespan and hence are useful for search space reduction from surveillance. Gender and age estimation are the most researched demographic attributes. Researchers estimate gender from face [1826], fingerprint [27-32], iris [33,34], body [35-40], hand [41-46] and speech [47-51]. Gender from face covers a major research segment and achieves $99 \%$ [22] classification accuracy. However, performance decreases dramatically for a realistic and unconstrained environment. It imposes illumination changes, occlusion, different views, and poses. Surveillance videos cover such unconstrained scenarios and it is also very difficult to acquire face, fingerprint, iris, hand, and speech biometrics from surveillance. Gender classification from the full human body is a more suitable way for surveillance applications.

Anthropometric measurements are a traditional way of body measurements. Since the era of Bertillonage $[1,2]$ they have been in security practices and are currently known as anthropometric and geometric soft biometrics. Facial landmarks related to chin, mouth, eyes, and nose are anthropometric mea- 
surements related to facial geometry [52-55]. Body height [56-59] is the most researched anthropometric attribute. Similarly, other body measurements, like torso length, leg length, and step length [58], are also useful. Medical characteristics help monitor a person [12] with the help of a computer vision-based technique. Bodyweight, body mass index (BMI), and skin quality are soft biometrics used for an application like early detection and prevention of diseases like skin cancer. Another class of soft biometrics is material and behavioural. It includes various accessories worn by a person like a hat, scarf, eyeglasses; different clothes and clothing colours; scars, marks, and tattoos on the body. Such soft biometrics also play a crucial role in retrieval. For example, a description, a male with a green t-shirt and blue jeans wearing a black cap and carrying a backpack, contains clothing colours, clothing types, accessories (i.e., cap and backpack) as soft biometrics. Material and behavioural attributes are highly time-inconsistent. E.g., a person wearing a white shirt and black pants in the morning may have different clothes (blue t-shirt and white shorts) in the afternoon of the same day. Thus, descriptions for morning and afternoon are different for person retrieval. Time consistency discussion is in Sec. 2.1.

\subsection{Characteristics of soft biometrics}

Research interest is now more inclined towards applications where person retrieval at a distance is necessary, based on ancillary information like soft biometrics [60-62]. Some early research [6,63-67] shows the use of soft biometrics to improve the performance of the primary biometric system by narrowing down the search space in the initial stages. Jain et al. [6] mentioned that soft biometrics are inexpensive to compute, with no person cooperation requirement, and derived at a distance. Similarly, various pros and cons of soft biometrics are in further discussion.

Registration free / enrollment free:

Traditional biometric systems require prior registration of the biometric signature for identification in the future. However, prior registration of soft biometric attributes of a specific person is not required. The training is offline, and the model is useful for retrieval of the individual.

Compatible with human language:

Traditional biometrics features like fingerprints are discriminative. However, they cannot be describable by linguistic labels. It creates a semantic gap between human beings and the person retrieval system. Soft biometrics is a form of biometrics that uses labels people generally use to describe or identify each other like tall, female, young and blond hair. They help to bridge the semantic gap by generating descriptions understandable by humans. 
Cost and computationally effective:

Face, fingerprint, palm print, and iris-like biometrics require dedicated sensors for acquisition and a constrained environment. Soft biometric attributes like height, gender, and clothing colours are extractable from single low-quality surveillance videos where the traditional biometric acquisition fails. It reduces costs on the overall system development.

Recognizable from a distance:

Soft biometrics attributes are identifiable from a distance, e.g., clothing colour, clothing type, gender.

No cooperation from the subject:

Soft biometrics attributes (e.g., clothing colour, gender) acquisition does not require any cooperation from the subject. Unconstrained and non-intrusive acquisition is also non-invasive, which makes it suitable for surveillance applications.

Privacy preservation:

Soft biometrics-based description (e.g., a tall female with a blue t-shirt) only provides partial information. Such descriptions are not unique to an individual. It offers solutions to privacy issues related to soft biometric data storing.

\section{Uniqueness:}

Soft biometric attributes are not unique to an individual, i.e., a single attribute (e.g., female) does not uniquely retrieve the person. Multiple soft biometrics together can help to overcome this limitation.

Permanence:

Soft biometrics are not permanent attributes of an individual like a fingerprint. It makes person retrieval more challenging.

\subsection{Structure of the paper}

The paper presents the use of soft biometrics for person retrieval and suggests their strengths and weaknesses. It recommends the most discriminative and robust soft biometrics during person retrieval under challenging conditions. Besides covering the general frame work for retrieval using textual query it also reviews and compares large-scale datasets. It further provides classification for retrieval methods and provides state-of-the-art reviews for soft biometric attribute-based retrieval methods. Further, the integration of benchmark datasets and methods allows an effective comparison of their performance. In this way, the presented review is entirely different from other soft biometrics basics reviews [9-12], which focus mainly on soft biometric attribute 
retrieval from the persons image. A review of recent datasets, state-of-theart retrieval methods including vision and natural language processing, and dataset-method integration is not available in the other review articles.

The contributions of the paper are summarised as follows:

1. Recommends the most discriminative soft biometrics under challenging conditions and show case their use for person retrieval.

2. Provides the most comprehensive coverage of soft biometrics datasets.

3. Spans the complete spectrum for state-of-the-art methods; image based, discrete attribute based and natural language description-based retrieval.

4. Integrates datasets and methods for quantitative and objective performance evaluation.

5. Discusses open research problems in person retrieval using soft biometrics and their possible solutions.

\section{Soft biometrics based person retrieval in surveillance videos}

Person retrieval has become the most critical and essential task for surveillance and security. Various government sectors and private organizations invest more in computer vision-based research techniques due to ever-increasing video-based data. It is observable that although the frame in Fig. 2(a) is at low resolution and captured at a distance, a detailed description can still be available. For example, one can get a narrative like a male with a green $t$-shirt and blue jeans wearing a black cap, and carrying a backpack. Such human description naturally contains soft biometrics like gender (male), clothing color (green, blue), clothing type (short sleeve), accessories (cap, backpack). It indicates that soft biometrics have a strong relationship with human descriptions. Thus soft biometric attribute-based person retrieval systems are more suitable for an unconstrained environment.

Computer vision has created enormous opportunities to develop an algorithm for intelligent video processing which is useful for retrieval. Developing such algorithms is also tricky due to various problems like video frame resolution, different camera views, poses, illumination conditions, occlusion, and background merging with the foreground.

2.1 Challenges for person retrieval in surveillance

\section{C1. Low-resolution videos:}

Surveillance videos have lower resolution since they have to be recording for long periods of time. Fig. 2 shows such a surveillance frame where it is challenging to extract face information from the low-resolution frame with the distance between the camera and the person. A face recognition-based retrieval 

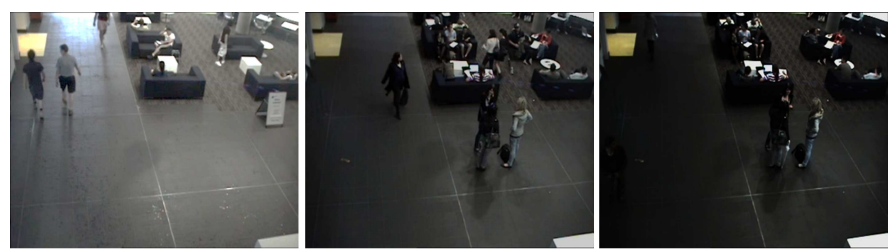

(a)
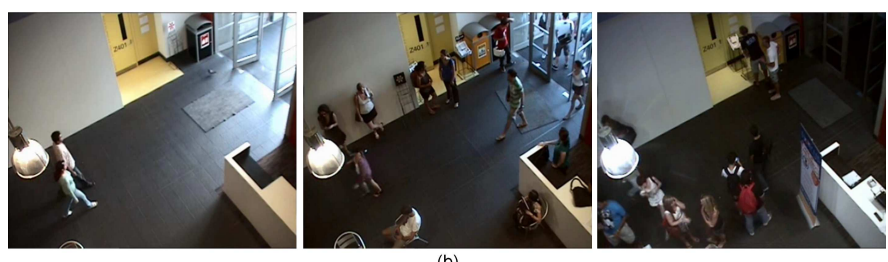

Fig. 4 Varying illumination conditions in multiple frames of the single camera. (a) Cam1 and (b) Cam4 in AVSS 2018 challenge II database [5]. Frames from left to right are with good to poor illumination.

system fails to establish identity in such challenging conditions. Also, the lowresolution structure does not help to extract useful features. Hence, it creates a problem for person retrieval.

\section{C2. Illumination changes / varying illumination conditions:}

The video looks good if the illumination is excellent. A proper lighting condition is the key to better visual information. Fig. 4(a) and (b) show singlecamera surveillance frames with different illumination conditions. Surveillance frames from left to right are with good to poor illumination conditions. People and their clothing colours are visible in the frames with proper lighting conditions. They are challenging to observe in reduced illumination and create a problem in person detection and colour classification.

\section{C3. Occlusion:}

Occlusion in the surveillance frame is a challenge where a person of interest is not completely visible. Fig. 5 shows such sample frames where a person of interest is within a green bounding box in each frame. It also creates issues for detection and the segmentation process in person retrieval. Occlusion also affects the estimation of an anthropometric attribute like height.

\section{C4. Merging with background information:}

Fig. 6 shows surveillance frames in which the person of interest merges with the background. It happens due to low illumination or similarity in clothing colour and background. Persons are not visible though they are not under occlusion in each frame. 

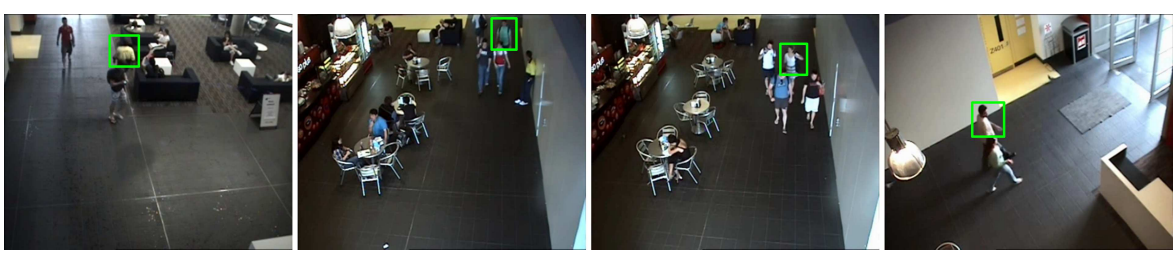

Fig. 5 Occlusion in surveillance frames [5]. The persons of interest are shown with green bounding box.
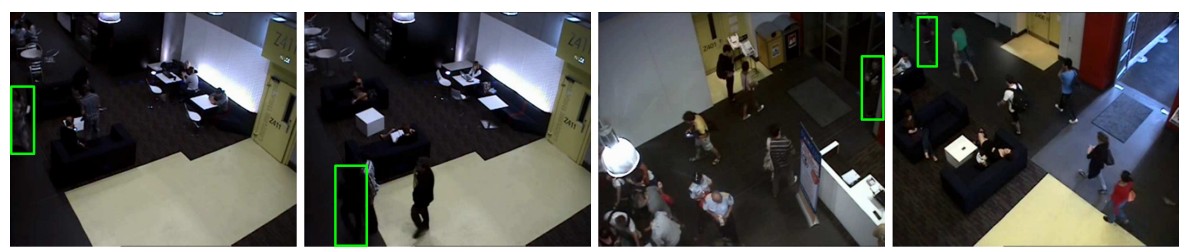

Fig. 6 Person of interest merges with background information in surveillance frames [5]. The persons of interest are shown with green bounding box.

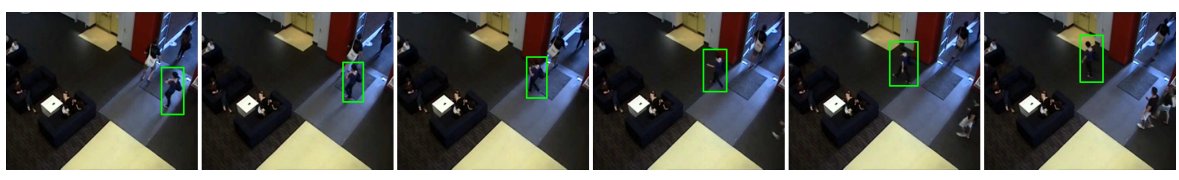

Fig. 7 Different lighting condition in a scene for a single sequence [5]. The person of interest is shown with green bounding box.

\section{C5. Different illumination in the scene for a single sequence:}

Fig. 7 shows a sequence of surveillance frames where illumination varies in different parts of the frame. The person shown within a green bounding box is moving from right to left in the frame. It shows that the outside light is entering the room in the top right corner of the scene. It is observable that the colour information keeps on changing as the person moves through such a region.

\section{C6. Different viewpoints of the same person:}

A person may appear with different poses in the same camera scene or scenes of multiple camera networks. Appearance-based methodologies expect minimal or no change in the visual appearance of the person. Fig. 8 shows a scenario where the pose of a person keeps on changing, and hence the appearance in the camera changes. Retrieval is difficult in such a system. Pose changes also create difficulties for methods that retrieve a person based on his or her gait.

\section{C\%. Crowded scene:}



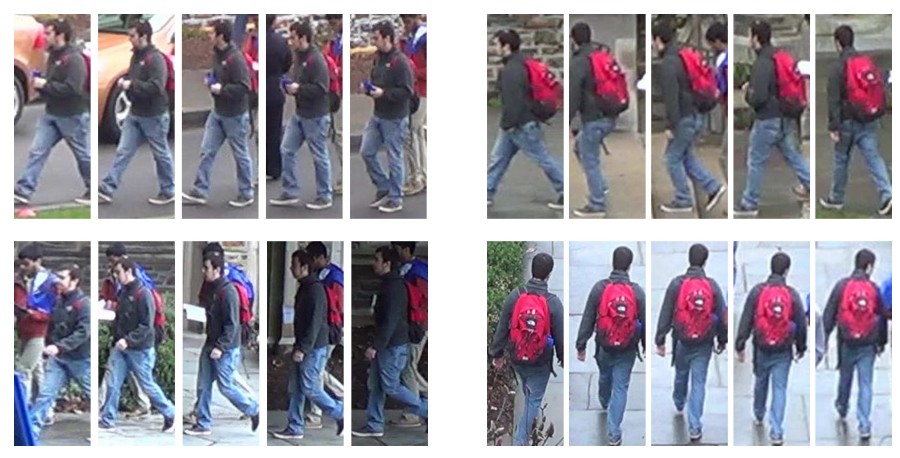

Fig. 8 Different viewpoints of same person [107].
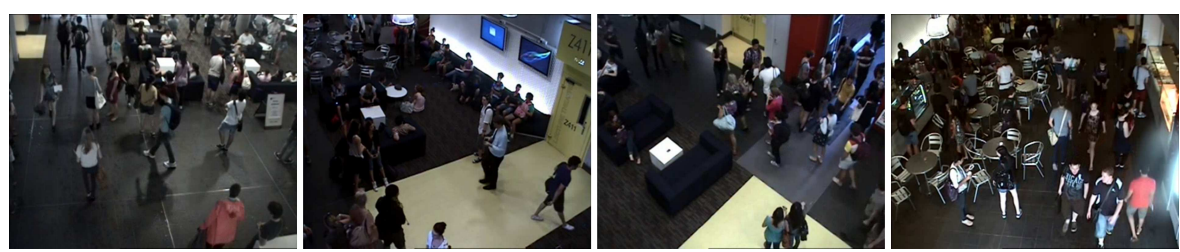

Fig. 9 Crowded scene [5].

Sitting areas, canteens, shopping malls, airports and railway stations are places where a massive crowd is observable. Such frames are in Fig. 9. A crowded scene contains occlusion and persons with a similar appearance cause difficulties in retrieval.

\section{C8. Indoor and outdoor environment:}

Surveillance cameras are at different indoor places (e.g., shopping malls, canteens) and outdoor places (e.g., university campus, public roads). Fig. 4, 5, 6, 7 and 9 show indoor environments, while Fig. 8 shows an outdoor environment. Both environments create different illumination conditions. Outdoor environments are affected explicitly by various weather conditions and daynight illuminations.

\section{C9. Time consistency / time duration:}

As discussed in Sect. 1.1, material and behavioural attributes like clothing colour and clothing type are highly time-inconsistent. The same person may have different clothing even on the same day, i.e., morning (white shirt and black pants) and afternoon (blue t-shirt and white shorts). Also, attributes like hairstyle and hair colour may change over the days, and age obviously varies over years.

C10. Type of camera / multiple cameras: 
Surveillance feeds captured from different types of cameras have other characteristics. For example, red clothing colour perception may differ from a bullet camera to an unmanned aerial vehicle (UAVs) camera. It creates many concerns for clothing colour-based retrieval.

\section{C11. Unavailability of a generic dataset:}

The available datasets (Sec. 2.4) do not cover all the challenges, e.g., a dataset may have only indoor environment images. Also, some of the soft biometric (e.g., clothing colour) annotation may be missing. Such limitations incur the challenge of developing a universal end-to-end system.

The conditions under which a surveillance network operates is challenging. These conditions are responsible for producing unsatisfactory results. The surveillance system should be robust to create a meaningful impact in the real world. It is crucial to select soft biometric attributes that help person retrieval under challenging conditions.

\subsection{Soft biometric attribute selection}

Soft biometric attributes are not unique; for example, there may be multiple people with a blue torso colour. Thus, it produces numerous matches for the given textual query. Therefore, it is advantageous to use the most discriminative attributes for person retrieval. Different soft biometrics have certain advantages, and they are discussed below. For example, a surveillance video may contain different view angles and distance. The person's height is invariant to such concerns $[68,69,78]$. Clothing colour is also one of the most discriminative attributes. It has the following advantages:

- Colour is more immune to noise.

- It is insensitive to dimension, view angle, and direction.

- Colour is recognizable from a far distance.

Clothing type is also insensitive to view angle. Gender is identifiable from near as well as far distance and different view angles. Table 1 shows the strength of soft biometric attributes against challenging conditions. " $\checkmark$ "indicates that a particular attribute is useful for the retrieval process against a specific challenging scenario. For example, a standing person's height estimation requires head and feet points $[68,69,78]$. The extraction of those points is not affected by different view angles, near or far fields, illumination, and low resolution. However, those points are non-extractable when a person is occluded or has a pose like sitting. Similarly, " $\times$ " indicates that a particular attribute is not useful for the retrieval process against the challenge. 
Table 1 Soft biometric attribute selection for person retrieval against different challenges.

\begin{tabular}{|c|c|c|c|c|c|c|c|c|c|}
\hline & View angles & Far field & Near field & Illumination & Partial occlusion & Pose & Low resolution & Time & Camera \\
\hline Height & $\checkmark$ & $\checkmark$ & $\checkmark$ & $\begin{array}{c}\checkmark \\
\end{array}$ & $x$ & $x$ & $\checkmark$ & & $\checkmark$ \\
\hline Cloth color & $\checkmark$ & $\checkmark$ & $\checkmark$ & $x$ & $\checkmark$ & $\checkmark$ & $\checkmark$ & $x$ & $x$ \\
\hline Cloth type & $\checkmark$ & $\checkmark$ & $\checkmark$ & $\hat{v}$ & $\times$ & $x$ & $\checkmark$ & $x$ & $\hat{x}$ \\
\hline Gender & $\checkmark$ & $\checkmark$ & $\checkmark$ & $\checkmark$ & 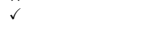 & $\checkmark$ & $\checkmark$ & $\checkmark$ & $\checkmark$ \\
\hline Torso pattern & $\checkmark$ & $x$ & $\checkmark$ & $\times$ & $x$ & $x$ & $x$ & $x$ & $x$ \\
\hline Body geometry & $x$ & $\checkmark$ & $\checkmark$ & $\checkmark$ & $x$ & $x$ & $\begin{array}{c}x \\
y\end{array}$ & s & a \\
\hline Hair (type \& color) & $\checkmark$ & $x$ & $\checkmark$ & $x$ & $x$ & $x$ & $x$ & $\times$ & $x$ \\
\hline Shoe (type \& color) & $\checkmark$ & $x$ & $\checkmark$ & $x$ & $x$ & $x$ & $x$ & $x$ & $x$ \\
\hline Accessories (bag, hat) & $x$ & $x$ & $\checkmark$ & $x$ & $x$ & $x$ & $x$ & $x$ & $x$ \\
\hline
\end{tabular}

\subsection{Vision based person retrieval system}

This section discusses a person retrieval system that uses a natural languagebased textual query. Researchers propose methods using handcrafted featurebased retrieval [61-67,70-77], deep learning feature-based linear filtering [68, 69,78], parallel classification of attributes [79-81] utilization of Natural Language Processing (NLP) algorithms to process textual queries [82-89]. Such a plethora of methods consists of person detection, segmentation, soft biometric attributes classification, and person identification as crucial steps in the person retrieval process. These key steps are given in Fig. 10 and discussed as follows:

Step-I (Person detection): Person retrieval in surveillance is a challenging task because such scenarios are usually in the wild, containing various objects (e.g., chair, table, car, and train) apart from the person. Hence, person detection is the critical initial step before textual query-based person retrieval. Person detection is the task of locating all persons in the surveillance frame. It was a foreground-background separation problem before the era of deep learning. A person is a foreground object, which is different from the rest of the background. Early research detected the person by different methodologies like background subtraction $[70,90]$, adaptive background segmentation [91], Gaussian Mixture Model (GMM) [92], and Histograms of Oriented Gradients (HoG) [93].

Deep learning-based methodologies are becoming popular in recent years due to their robust feature extraction and learning ability. The computer vision community considers person detection as an object detection problem. Some of the popular object detection frameworks are "You Only Look Once (YOLO)" [94], Single-Shot Multibox Detector (SSD) [126], Region-based Convolutional Neural Network (R-CNN) [95], Fast R-CNN [96], Faster RCNN [97] and Mask R-CNN [98]. Person detection provides a bounding box for each person in the frame.

Step-II (Segmentation): Segmentation follows person detection. Segmentation can be either in the form of a body part from a bounding box or semantic segmentation within the bounding box. Such segmentation is shown in Fig. 11. A full-body can be segmented into three major parts: head and shoulders, upper body, and lower body (see Fig. 11(a)). Fig. 11(b) shows semantic segmentation in which each pixel belonging to the person within 


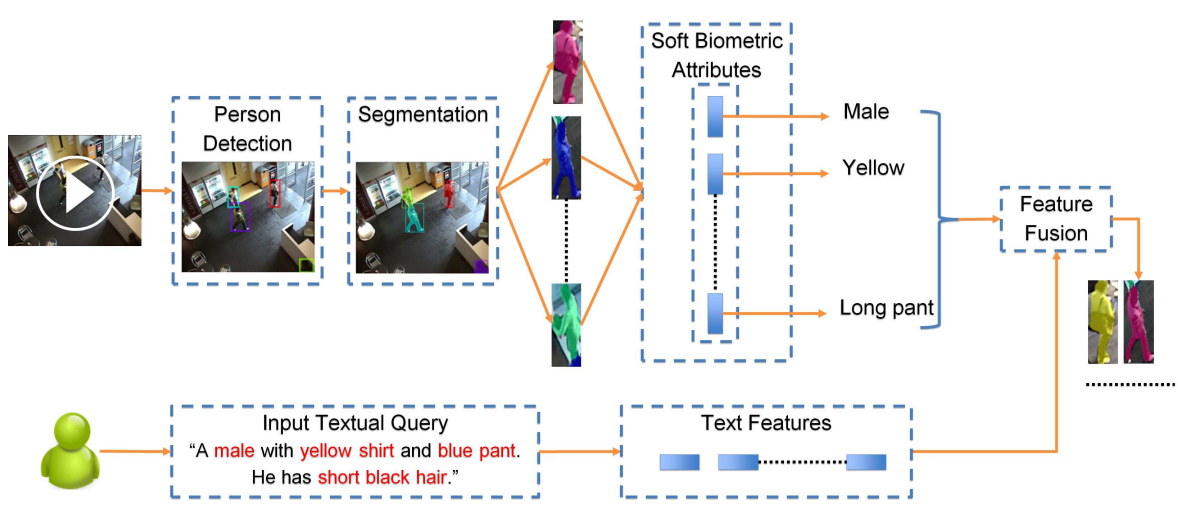

Fig. 10 Person retrieval in surveillance using textual query.

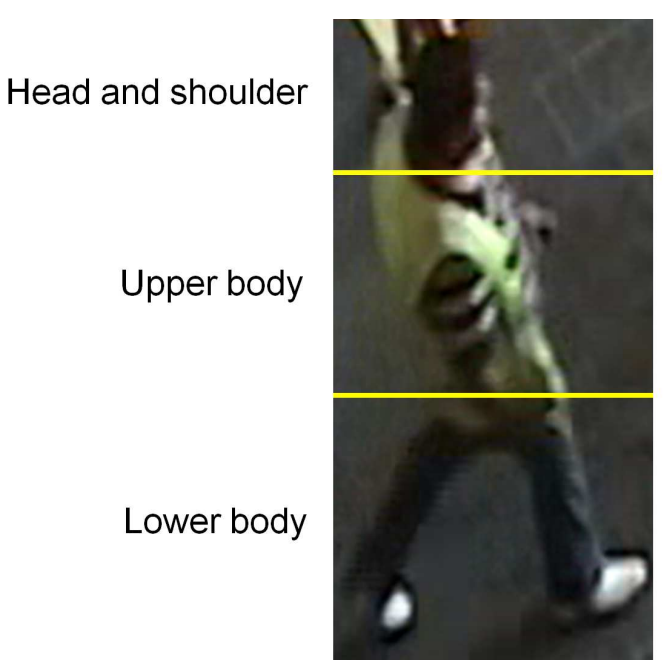

(a)

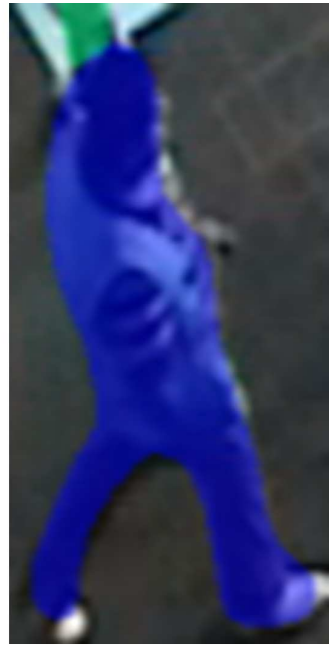

(b)

Fig. 11 Segmentation: (a) body part segmentation and (b) semantic segmentation.

the bounding box has a label. Mask R-CNN [98] provides both person detection and semantic segmentation together.

Step-III (Soft biometric attribute recognition): Full-body images and segmentation outputs are supplied to extract soft biometric attributes. Full-body images are useful for extracting characteristics like height and gender $[68,69,78]$. On the other hand, clothing colour, clothing texture, clothing type, shoes, hair, and accessory attributes are available from different body parts. Usually, details from video frames are visual features. Recent development shows multi-attribute learning for person attribute recognition. Researchers propose various networks like Deep learning-based Multiple Attribute Recognition (DeepMAR) [152], Attribute Convolutional 


\section{Gender: Female Torso type: Short Sleeve Torso color: Black Torso texture: Plain Leg type: Short Leg color: White Luggage: Yes}

(a)

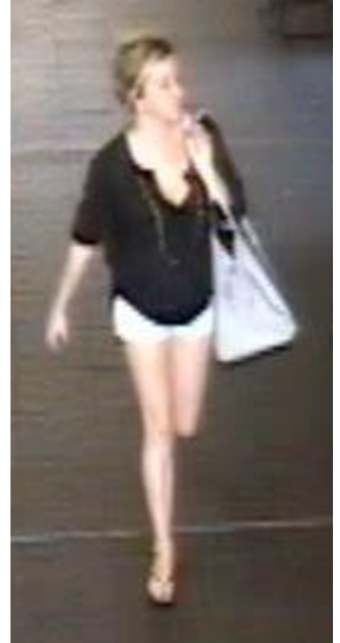

The woman has light brown hair. She is wearing a short sleeve black top with white short and carrying a purse in left hand.

(b)

Fig. 12 Textual query: (a) discrete attribute query and (b) natural language description

Net (ACN) [153], and Multi-Label Convolutional Neural Network (ML$\mathrm{CNN})[154]$.

Step-IV (Text feature extraction): Textual attribute query or natural language description is another input to the person retrieval system. Such textual query samples are given in Fig. 12. Textual attribute query (Fig. 12(a)) is cheaper to collect in terms of attribute wise information and has a less complicated sentence structure in comparison with natural language description (Fig. 12(b)). The data is collected separately for all attributes which is useful in the retrieval process. For example, torso type attribute value is available from one of the predefined classes \{Long Sleeve, Short Sleeve, No Sleeve\}. However, such a discrete attribute query has a fragile appearance, descriptive ability, and practical usage limitation.

In contrast to textual attribute query, natural language description has more complex sentence structures. However, it provides a detailed description of the person. It is essential to extract relevant information from such a human verbal description. For example, the description in Fig. 12(b), 'women', 'brown hair', 'blacktop'are more relevant information than 'the', 'a', and 'has'. Such relevant information forms textual features. NLP based algorithms help to extract textual features from natural language descriptions.

Step-V (Feature fusion and retrieval): Visual features for each person are available from surveillance videos, and textual features are extractable from the textual query. Person retrieval from surveillance using textual query covers two major problem domains: (i) computer vision and (ii) natural language processing. Hence, cross-modal feature embedding is applica- 
ble in the feature fusion block (see Fig.10). Finally, a person(s) matching the textual query is retrieved. There is a possibility of multiple person retrieval as soft biometrics are not unique to an individual.

The accuracy of person retrieval depends on the complexity of the training and testing data sets. The retrieval robustness relies on the availability of the richly annotated data sets. The performance evaluation of the method is done by evaluation metrics like accuracy, True Positive Rate (TPR) and Intersection-over-Union (IoU). Therefore, the following sub-sections discuss person retrieval datasets (available in the public domain) and evaluation metrics.

\subsection{Person retrieval datasets}

A variety of challenging datasets are an essential entity for any research and development task. Datasets with extensive annotations and reliable evaluation strategies help to create a robust algorithm. Researchers have developed many public datasets for person identification and re-identification in the past two decades. Table 2 shows only datasets with soft biometric annotations, which help create textual attribute query or natural language description for person retrieval.

Dataset comparisons have the following parameters: number of persons, number of images, resolution of the image (width $\times$ height)), number of soft biometrics annotations, number of cameras used to collect the dataset, type of attribute query, challenges covered, and whether the dataset is with full surveillance frame or cropped person. Abbreviations for challenges and types of attributes are in Table 2, which are useful for further discussion. Layne et al. [102] annotate the VIPeR [99] dataset with 15 soft biometric attributes like shorts, skirt, sandals, backpack, jeans, logo, v-neck, stripes, sunglasses, openouterwear, headphones, long-hair, short-hair, gender and carrying an object. These are binary attributes e.g., gender has value from set $\{0,1\}$, where $0=$ male and $1=$ female.

Zhu et al. [110] introduce the Attributed Pedestrians in Surveillance (APS) database specifically for pedestrian attribute classification, which can also be useful for person retrieval. The APS consists of 11 binary attributes and two multi-class (total 35) attributes, i.e., clothing colour of upper-body and lowerbody. APS is the first dataset to use clothing colour and covers more challenges compared to VIPeR. Layne et al. [103] further extend annotations for VIPeR [99], PRID [100], and GRID [101] from 15 to 21 by introducing shirt colour (red, blue, green), shirt shade (light and dark) and pant and hair colour. New datasets like VIPeR and PRID are having fixed image resolution, limited cameras, C2, C5, and C8. GRID upgrades such parameters and introduces $C 1$ and C3. However, it is limited to some images. VIPeR, PRID, and GRID contain photos from outdoor scenes only. 


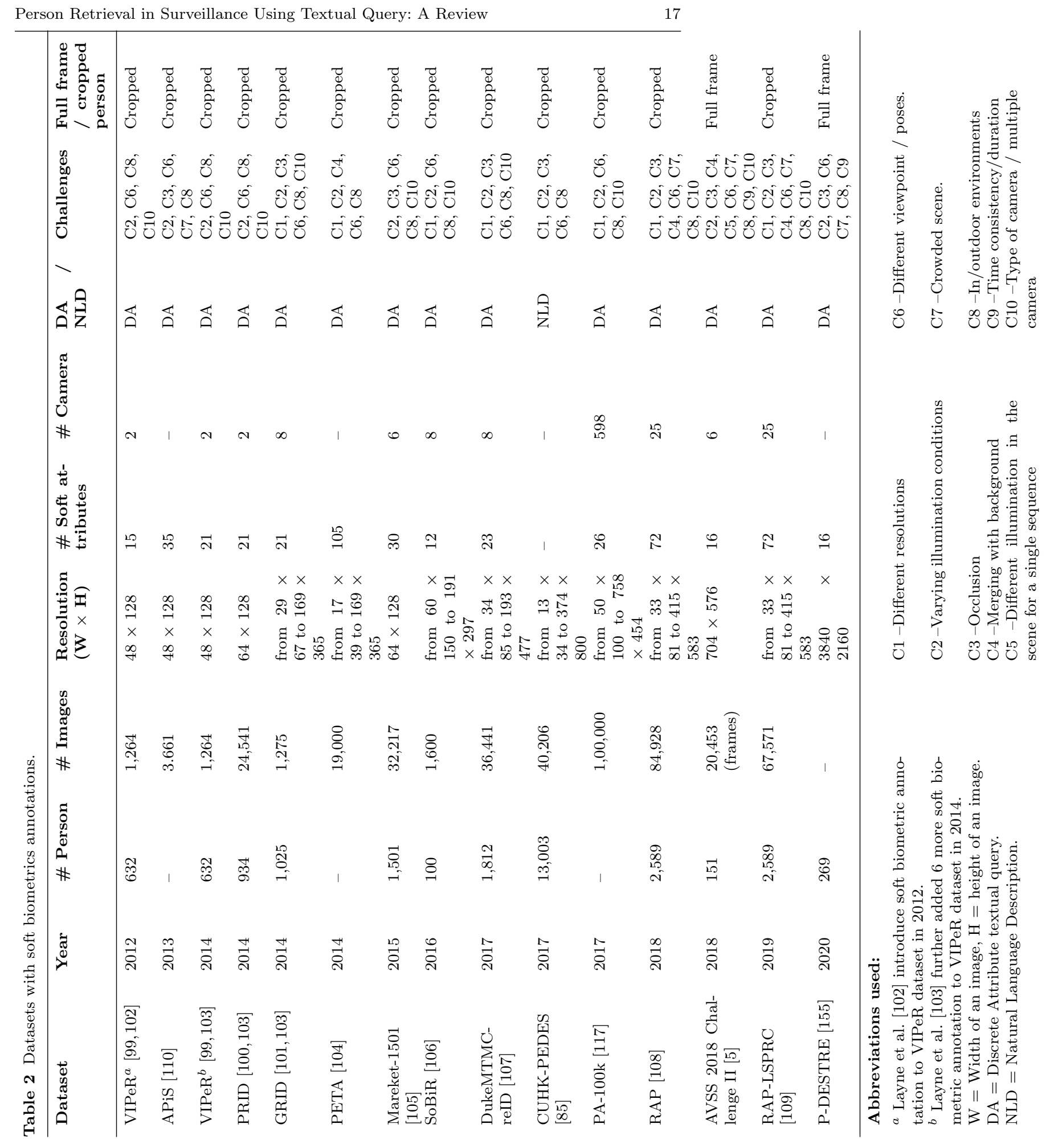


Deng et al. [104] introduce the first large-scale PEdesTrian Attribute (PETA) dataset to overcome the limitations of VIPeR, APS, PRID, and GRID datasets. They introduce $C 1, C 2, C 4, C 5, C 7, C 8$ and diversity by annotating 61 binary and 4 multiclass (total 105) attributes in 19,000 images collected from surveillance datasets like 3DpeS [111], CAVIAR4ReID [112], CUHK [113], GRID [101], i-LIDS [114], PRID [100], SARC3D [115], TownCentre [116] and VIPeR [99]. Martinho et al. [106] create the SoBiR dataset with 12 multi-class attributes. It is a comparatively smaller dataset with 1,600 images. However, each soft biometric attribute possesses multiple classes which help discriminate against people better.

Recent research advancement is increasing rapidly in deep learning domains, which requires a more diverse and huge amount of data for better generalization. Datasets like VIPeR, APS GRID, and SoBiR are too small for deep learning-based frameworks. Datasets published in the last five years provide a good amount of data to create robust models and algorithms. Large-scale datasets such as Mareket-1501 [105] have 32,217 images of 1,501 persons and DukeMTMC-reID [107] provides 36,441 images of 1,812 persons. These small and large-scale datasets use discrete attribute-based queries (Fig. 12(a)), which has limited practical usage, as discussed in Sect. 2.3. Thus, Li et al. [85] propose a large-scale dataset with natural language description-based annotations. The dataset is known as CUHK Person Description Dataset (CUHK-PEDES). It provides 40,206 images of 13,003 persons with 80,412 description sentences. It is the only large-scale dataset with a natural language description that provides an opportunity to explore the relationship between language and vision for a person retrieval problem.

Liu et al. [117] propose a large-scale pedestrian attribute (PA) dataset with 100000 images. It is known as PA-100k. It consists of the highest number of images in all the datasets with a more diverse collection from 598 scenes. It can only be used for attribute-based person retrieval, not for person reidentification. Further increasing the scale in all aspects, Li et al. [108] created a Richly Annotated Pedestrian (RAP). Li et al. [109] also make a RAP Large-Scale Person Retrieval Challenge (RAP-LSPRC) dataset. RAP-LSPRC is a subset of RAP with a comprehensive evaluation and benchmark dataset. By far, RAP contains the highest number of images $(84,928)$ collected from 25 surveillance cameras installed in the unconstrained indoor environment of a shopping mall. RAP and RAP-LSPRC are useful for both person retrieval and person re-identification tasks.

Datasets discussed so far contain an image gallery of cropped persons from surveillance video frames. Hence, in such image problems related to person detection, occlusion, merging with background, crowded scene, and illumination variations in a single sequence cannot be overcome. It limits the development of an end-to-end intelligent surveillance system. Halstead et al. first developed an AVSS 2018 challenge II dataset with full surveillance frames $(20,453)$ collected from 6 indoor cameras. It is the only dataset that contains video sequences of 151 persons (110 training +41 testing) with varying surveillance frames from 21 to 290 . They provide shallow resolution $(704 \times 576))$ frames. 
They cover most real-time surveillance challenges (Sect. 2.1) to develop an end-to-end solution for person retrieval. Such surveillance frames are in Fig. 4, 5, 6, 7, and Fig. 9 .

Recently, Kumar et al. [155] released a UAV-based dataset for Pedestrian Detection, Tracking, Re-Identification, and Search (P-DESTRE) from aerial devices. It also contains full surveillance frame videos. These videos are captured with $4 \mathrm{~K}$ spatial resolution $(3840 \times 2160)$ and over different days and times. Thus, P-DESTRE is the first dataset to provide consistent ID annotations across multiple days. Other datasets discussed so far do not cover this challenge. Although this dataset covers rich annotations, it lacks clothing colour annotations. By reviewing all the datasets, the commonly used soft biometric attribute set is gender, upper-body cloth type, lower-body cloth type, upper-body cloth color, lower-body cloth color, upper-body cloth texture, clothing style, height, hair color, hairstyle, backpack, carrying an object, eye-wear, shoe\}. Such variety and diversity show the continuous evolution of challenging datasets proposed by active researchers in the vision and language fields.

\subsection{Evaluation metric}

Different performance measures evaluate the performance of any methodology. The section covers such evaluation metrics before discussing different procedures (Sec. 3) because knowledge of metrics helps to understand the performance of the method better.

Accuracy (ACC):

Classification accuracy usually evaluates machine learning models. It is the ratio of correct predictions to the total number of samples in the test. This metric represents the overall efficiency of the system. The metric works well with a balanced dataset, i.e., the number of positive and negative samples in the dataset are equal. The accuracy equation is given by eq. 1 ,

$$
A C C=\frac{T P+T N}{P+N}=\frac{T P+T N}{T P+T N+F P+F N}
$$

Where,

$\mathrm{P}=$ The number of real positive samples in the data

$\mathrm{N}=$ The number of real negative samples in the data

$\mathrm{TP}=$ True positive i.e., correctly identified

$\mathrm{FP}=$ False positive i.e., incorrectly identified

$\mathrm{TN}=$ True negative i.e., correctly rejected

$\mathrm{FN}=$ False negative i.e., incorrectly rejected

Let us consider an example of gender classification for 100 persons to understand the metric and the same example is going to be used for all the metric discussions in this section. Here, the positive class is male and negative class is female. 
Gender classification example:

\section{True positive (TP):}

Class: Male

Model predicted: Male

Number of TP results: 1

False Negative (FN):

Class: Male

Model predicted: Female

Number of FN results: 10

\section{False Positive (FP):}

Class: Female

Model predicted: Male

Number of FP results: 1

True Negative (TN):

Class: Female

Model predicted: Female

Number of TN results: 88

$$
A C C=\frac{1+88}{1+88+1+10}=0.89
$$

Accuracy is $89 \%$ i.e., 89 correct predictions out of 100 persons. Out of 100 persons, 11 are male, and 89 are female. 88 females out of 89 are correctly identified. But, only one male out of 11 is correctly identified. Thus, $89 \%$ of model accuracy seems very good. But accuracy alone as a metric can't help such a class-imbalanced problem as it gives a false sense of high accuracy. A real problem can arise when the cost of misclassifying minor samples is very high. Thus, the metric discussed below is helpful, along with accuracy.

\section{Precision:}

It is a positive predictive value and decides the fraction of correct positive predictions in total positive predictions. Thus, it provides the proportion of positive predictions of the samples that are correct. It is a measure of quality, and its high value means that the classifier returns more relevant results, i.e., it suggests how many selected items are appropriate. Precision is calculated as eq. 2 ,

$$
\text { Precision }=\frac{T P}{T P+F P}
$$

For gender classification example,

$$
\text { Precision }=\frac{1}{1+1}=0.5
$$

The model has precision of 0.5 i.e., $50 \%$ of the time the model is correct when it predicts the person gender as male.

Recall or True Positive Rate (TPR):

It represents the classifier's sensitivity, and it is the fraction of correct positive predictions to the total number of relevant samples. It provides the proportion of positive predictions in the correctly retrieved samples. It is a measure of the quantity and suggests how many pertinent samples have been selected. Recall is calculated as eq. 3 ,

$$
\text { Recall or } T P R=\frac{T P}{T P+F N}
$$



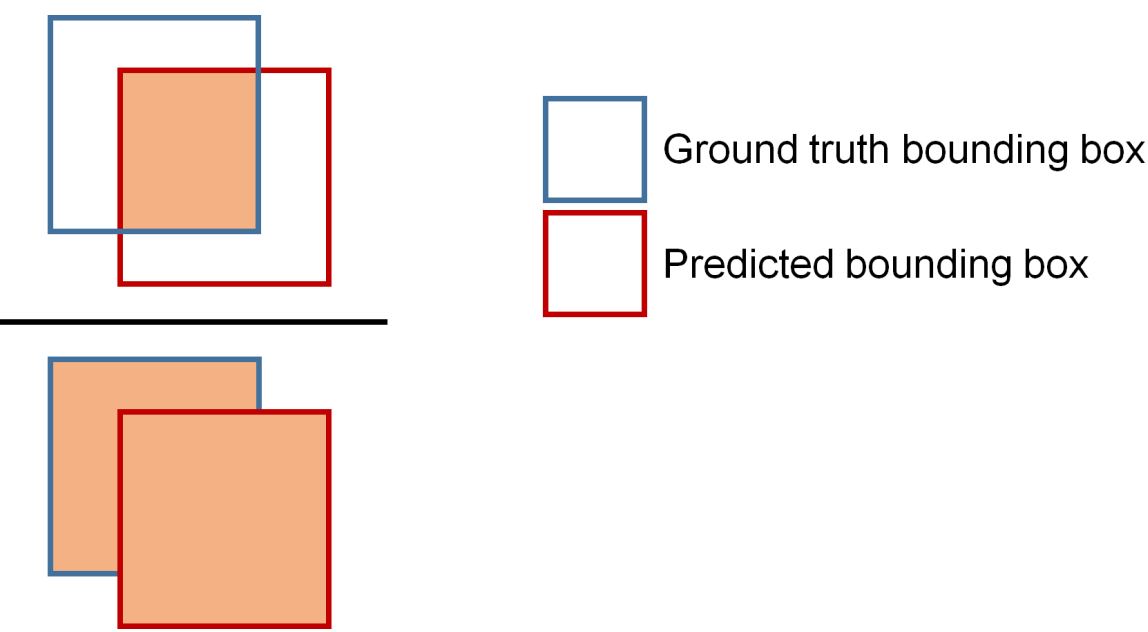

Fig. 13 Intersection-over-Union (IoU).

For gender classification example,

$$
\text { Recall }=\frac{1}{1+10}=0.09
$$

The model has a recall of 0.09 i.e., it correctly identifies $9 \%$ of total male persons.

Mean Average Precision ( $m A P)$ :

It is an average of maximum precisions at different recall values. An object detector accuracy is measured by mAP. It is calculated as eq. 4 ,

$$
m A P=\frac{1}{\text { Total Samples }} \sum_{\text {Recall }_{i}} \operatorname{Precision}\left(\text { Recall }_{i}\right)
$$

Intersection-over-Union (IoU):

The localisation accuracy of the object detector is measurable by Intersectionover-Union (IoU). It uses the bounding box of the object for evaluation. IoU is the fraction of the intersecting area to the union area between the output bounding box and the ground truth. It measures how good the detector is in localizing objects to the ground truth. Fig. 13 depicts the IoU metric.

$$
I o U=\frac{\text { Area of overlap }}{\text { Area of union }}=\frac{D \cap G T}{D \cup G T}
$$

$D=$ bounding box output of algorithm and $G T=$ ground truth bounding box.

Different person retrieval methodologies reviewed below use the evaluation metrics discussed above. Methods in [69,74-76,78-80] use IoU or average IoU, $[69,70,78]$ use TPR and $[81,82,84,85,89,134-139]$ use Top-1 accuracy as evaluation metrics. 


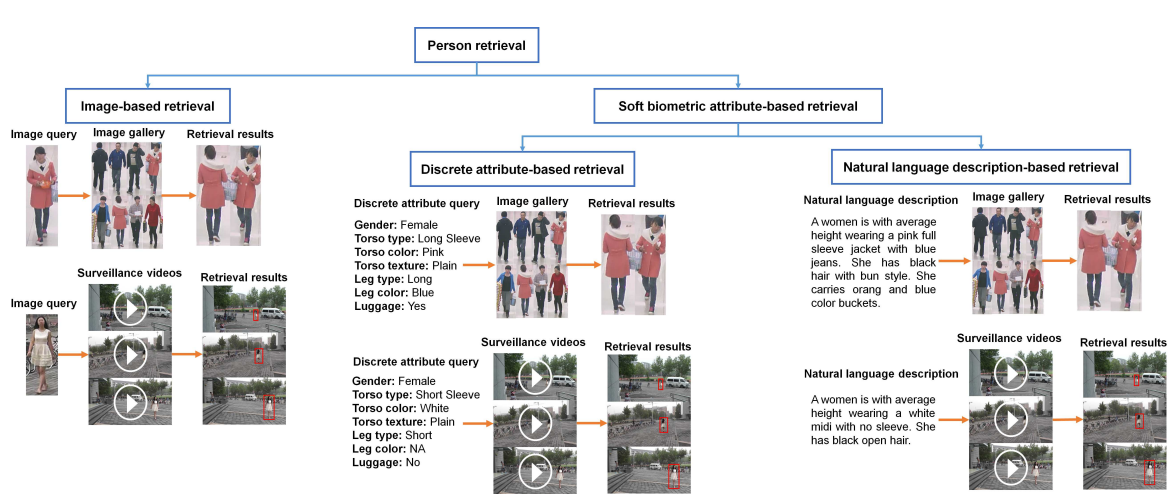

Fig. 14 Classification of person retrieval methodologies based on the type of input query. Each classification category shows example of person retrieval from cropped person image gallery and full frames of surveillance video. Images shown in image gallery are adopted from RAP [108] dataset and surveillance video frames are adopted from PRW [118] dataset to showcase the example.

\section{Person retrieval methodologies}

Person retrieval is a subject undergoing intense study for the past two decades due to its prime application to public safety. A large-scale intelligent surveillance system requires person retrieval using visual attributes or available person image(s). Vision community researchers are proposing a plethora of methodologies for robust person retrieval. They have two major categories based on the type of input query:

- Image-based retrieval.

- Soft biometric attribute-based retrieval.

Classification of person retrieval methodologies based on the type of input query is shown in Fig. 14. A person is retrievable from the cropped person image gallery or full frames of surveillance video. The latter is more suitable for real-time scenarios. The person retrieval example of each category is also shown in Fig. 14.

Image-based person retrieval methodologies aim to retrieve the target from the large-scale image gallery. This large-scale image gallery or dataset is from different non-overlapping cameras. Such person retrieval techniques are known as person re-identification (Re-ID). These techniques require at least one image of the target person for querying the system to perform re-identification of the same identity from different cameras. A person ReID technique assumes that the image gallery contains at least one image of the target person captured from a camera other than the query image. Such methodologies fail when a probe image is not available.

In contrast, soft biometric attribute-based person retrieval methodologies do not require a probe image. It uses a semantic person description generated from soft biometric attributes. Such a description (textual query) is input to the person retrieval system. Soft-biometric attribute-based retrieval is further 
divisible into two categories based on the type of textual query (Fig. 12 and 14):

1. Discrete attribute-based retrieval.

2. Natural language description based retrieval.

A discrete attribute-based person retrieval system searches for the person using soft biometrics attributes, e.g., clothing colour, clothing type, and gender. While a natural language description-based system accepts a natural description, e.g., A woman is with average height wearing a white midi with no sleeve. She has black open hair.

Applications like obvious question answering, image captioning, and person retrieval are gaining much attention due to the association of two widely researched domains, i.e., computer vision and natural language processing. Such applications expect the learning of discriminative feature representations from both images and text. The person retrieval algorithm ranks gallery images or person(s) in the surveillance frame according to their relevance to the description. The best matching images from the gallery or surveillance frame return the person of interest. This class of surveillance problems is known as text-based person search or person retrieval using natural language descriptions $[85,139]$. Such a person retrieval problem aims to enable retrieval of the person of interest using two different domain modalities, i.e., text and image. It takes one type of data as an input query (i.e., text). It retrieves the relevant data of another type (i.e., person image) by mapping textual and visual features (Sec. 2.3). Such kind of retrieval is also known as cross-modal retrieval [139-142].

Among all person retrieval methodologies, image gallery-based techniques are not preferable for end-to-end system-level evaluations. They do not consider challenges like occlusion, pose, and illumination in person detection from the full surveillance frame. Natural language description-based person retrieval systems are more suited for real-time person retrieval from surveillance videos. A person retrieved using a textual query is an input query to image-based person ReID systems for re-identification of the same target in the camera network. This paper discusses soft biometric attribute-based person retrieval methodologies in a further section.

\subsection{Discrete attribute-based person retrieval}

Soft biometric attributes like clothing colour and clothing type do not stay consistent for a given individual for a length of time. Such soft attributes keep on changing over a short period. Thus, soft biometric attribute-based person retrieval methods are short-term retrieval methods [71,119]. Such methods are well suited for applications like criminal investigation and searching for missing persons for a limited period. Discrete attribute-based person retrieval methods are further divisible into two categories based on how the features are extracted, i.e., 

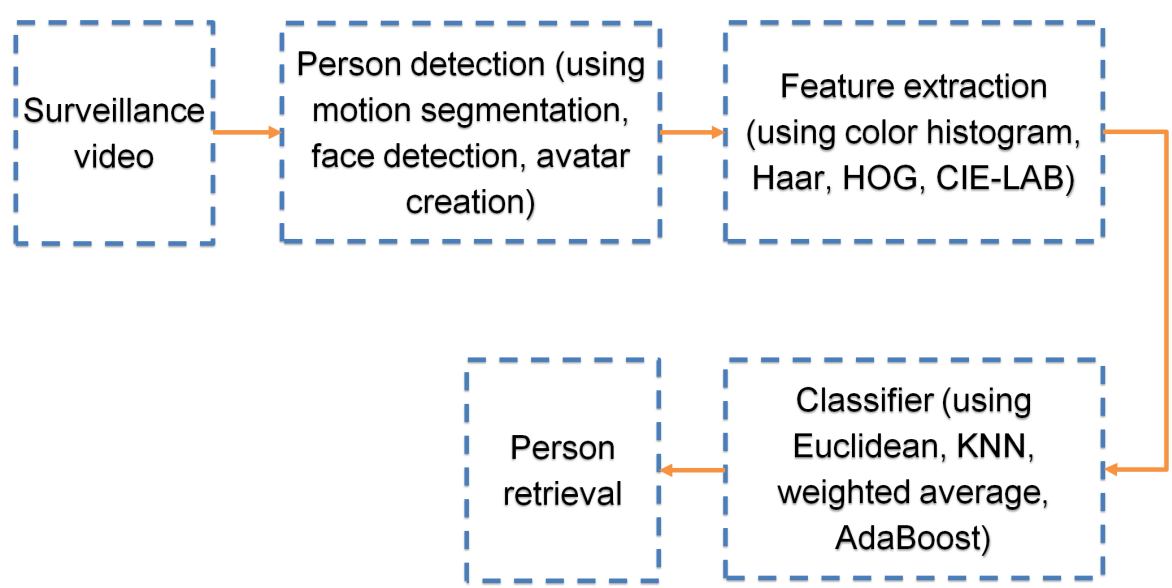

Fig. 15 Block diagram for handcrafted feature-based methods.

1. Handcrafted feature-based person retrieval.

2. Deep feature-based person retrieval.

\subsubsection{Handcrafted feature-based methodologies}

Research before the era of deep learning shows promising methods for person retrieval based on handcrafted features. Fig. 15 shows the general block diagram for person retrieval methods that use handcrafted features. Person detection is done using adaptive background segmentation, face detection, frame differencing, and query-based avatar creation. Feature extraction is a critical step where hand-engineered features are extracted using popular algorithms. Further, the feature fusion and classification are done for target person retrieval.

Vaquero et al. [71] exploit the limitation of the sensitivity of face recognition technology against illumination changes, low-resolution videos, and pose variations. They are the first to implement a video-based visual surveillance system that uses a persons fine-grained parts and attributes. Person detection uses a face detector. Further, the body is divisible into three regions with soft biometrics from the areas, i.e., face (hair type, eyewear type, facial hair type), torso (clothing color), and leg (clothing color). The nine facial attributes are extracted by training an individual Viola-Jones detector using Haar features. A normalized colour histogram is in hue, saturation and luminance (HSL) space for each body part (i.e., torso and leg).

Denman et al. [73] also propose a body part segmentation-based approach that detects a person using adaptive background segmentation [91] and segments the body into three parts (head, torso, and leg) using an average gradient across rows of the image. Calculation of height uses the Tsai [120] camera calibration approach and colour histograms for clothing colour. These features are fused using a weighted sum fusion approach. The algorithm evaluation on 
the PETS [121] dataset achieves the best results with an Equal Error Rate (EER) of $26.7 \%$ (colour model), the worst EER rate of $39.6 \%$ (size model), and an EER rate of $29.8 \%$ (a combination of colour and size).

The methods in $[71,73]$ detect a person first and then match the query rather than searching the person based on the query. Such limitation is removable by creating an avatar of the person from discrete soft biometric attributes [74]. The particle filter is then applied to drive a search in the surveillance frame. The methods mentioned here propose a new dataset for soft biometricbased person localisation. The height of a person is broken up into 5 classes (concise, short, average, tall, and very tall) and colour into 11 classes (black, blue, brown, green, grey, orange, pink, purple, red, yellow, and white). Localisation accuracy is measurable using Intersection-over-Union (IoU). These methods achieve $24.28 \%$ average localisation accuracy.

Pedestrian semantic attribute-based ApiS dataset is in [110] with 11 binary attributes and two multiclass attributes. AdaBoost classifiers are useful for binary attribute classification, and K Nearest Neighbours (KNN) classifier is useful for multiclass attribute classification. This paper focuses on attribute classification and is useful for person retrieval. Halstead et al. [75] further extend the surveillance dataset of [74] by annotating 12 multiclass attributes on 110 persons. The authors improve the performance of [74] by adding more attributes for avatar creation and considering the unequal reliability of each attribute. A 21\% improvement is reported over the baseline [74]. Avatar further extends in the form of channel representation (CR) [76]. The algorithm also incorporates shape information in the form of HOG representation to improve the performance. CR captures the spatial characteristics of colour and texture by representing them in the form of a multi-dimensional image. Each channel in CR represents either colour or texture information. The CR based approach achieves $44 \%$ average localisation accuracy.

Martinho et al. [106] generate a feature vector of length 4704 for each 256 $\times 256$ pre-processed image. They use CIE-LAB, and the Gabor channel filters feature for the generation of an Ensemble of Localised Features (ELF) descriptor. A HOG based descriptor is also useful for the creation of 2304 features. The Extra-Trees (ET) supervised ensemble learning algorithm is applied for classification. The algorithm achieves $12.5 \%$ and $20.1 \%$ at rank-1 accuracy for one-shot and multi-shot identification. Shah et al. [70] use clothing colour and clothing type for person retrieval. They detect the person using motion segmentation based on frame differencing. Background clutter is removable by a morphological operation. The ISCC-NBS colour model [122] and CIEDE2000 [123] distance metrics are useful for colour classification. Their method achieves a $67.96 \%$ TP rate, which is better than the GMM (44.88\%) approach. Table 3 shows an overview of the handcrafted feature-based methodologies. The performance column shows the highest value reported in the relevant literature in multiple scenario-based analyses. 


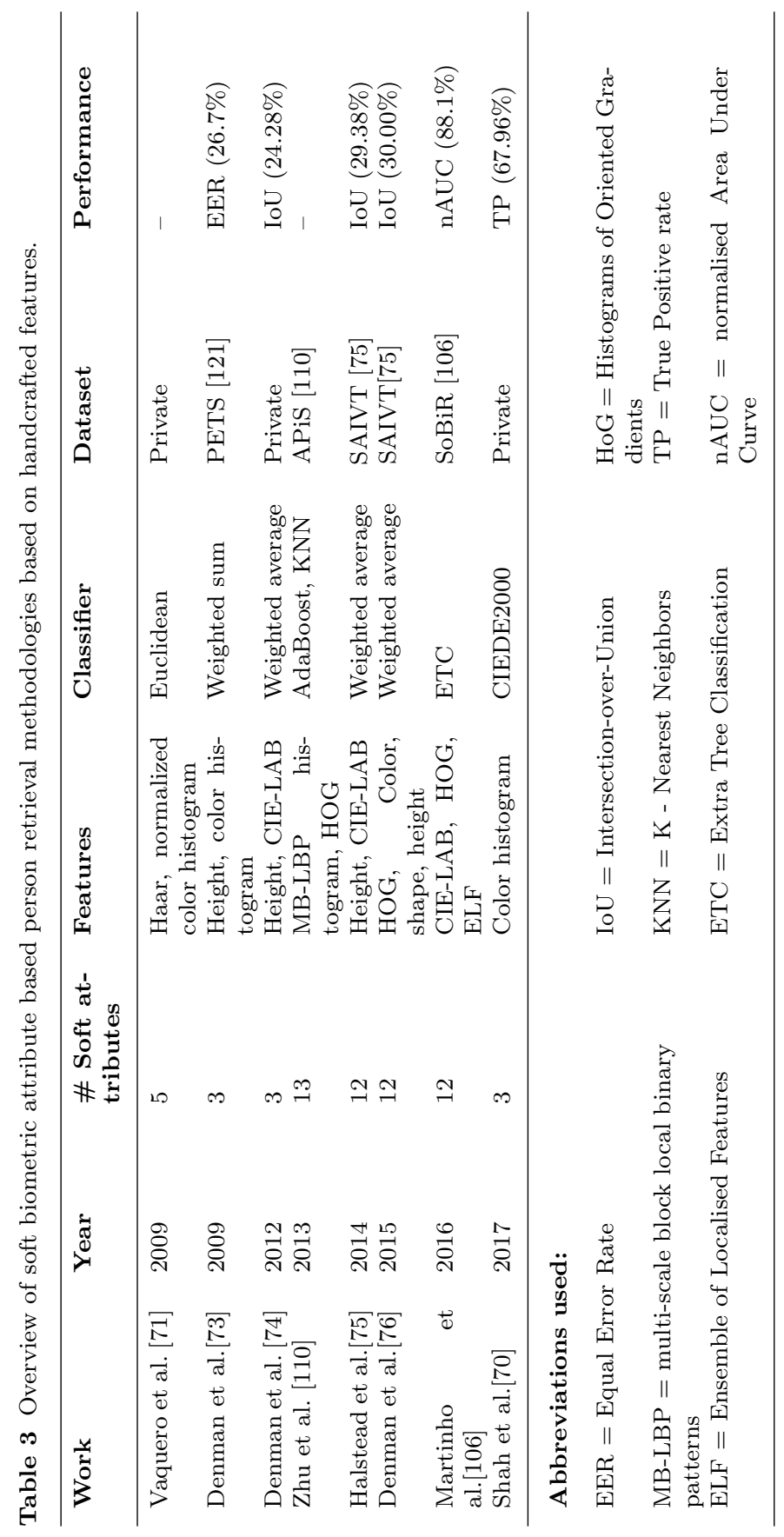


With the advent of deep learning techniques, person retrieval methods have seen a significant improvement in accuracy measures. Most state-of-the-art person retrieval methods are based on deep learning architectures.

\subsubsection{Deep feature-based methodologies}

Deep learning-based methodologies are becoming popular in the past few years due to their efficient feature learning ability. The deep Convolutional Neural Network (DCNN) based approach has gained more attention in the computer vision community. Table 4 shows an overview of the deep feature-based person retrieval methodologies. The performance column shows the highest value reported in the relevant literature in the case of multiple scenario-based analyses. Semantic Retrieval Convolutional Neural Network (SRCNN) developed by Martinho et al. in [124] shows the evaluation of a similar setup of [106]. Binary Cross-Entropy (BCE) and Mean Squared Error (MSE) loss functions quantify binary classification and regression. SRCNN achieves $35.7 \%$ and $46.4 \%$ at rank-1 accuracy for one-shot and multi-shot identification, respectively. Thus, a deep feature based SRCNN approach demonstrates a rank-1 accuracy improvement of $23.2 \%$ and $26.3 \%$ over a handcrafted feature-based system of [106].

The baseline method [76] of AVSS 2018 challenge II [5] dataset is implementable using handcrafted features, while all participants $[69,79,80]$ of the challenge have evaluations based on deep features. The following discussions in this section consist of methodologies implemented on AVSS 2018 challenge II [5] dataset as well as some other approaches on large scale datasets like Market-1501 [105], DukeMTMC [107], PA100K [117] and CUHK03 [113].

Galiyawala et al. [69] use height, clothing colour, and gender for person retrieval. Person detection and semantic segmentation using Mask R-CNN [98] help to remove the cluttered background. It results in a clutter-free torso patch for efficient colour classification. Height is available using a camera calibration approach [120]. Torso clothing colour and gender are classified using a fine-tuned AlexNet [125] based individual model. Sequential implementation of height, colour, and gender filter aims to eliminate the detected persons and leave only the target person. This linear filtering-based approach achieves an average IoU of 0.36. Schumann et al. [80] detect the person using the SingleShot Multibox Detector (SSD) [126]. Early-stage false positives are eliminated by background modelling based on a mixture of Gaussian distribution. The strategy of the ensemble of classifiers is adapted, and predictions are fused by computing mean or weighted mean. The evaluation is based on the Euclidean distance between a query vector and each detected person's attribute probability to produce the final result. 


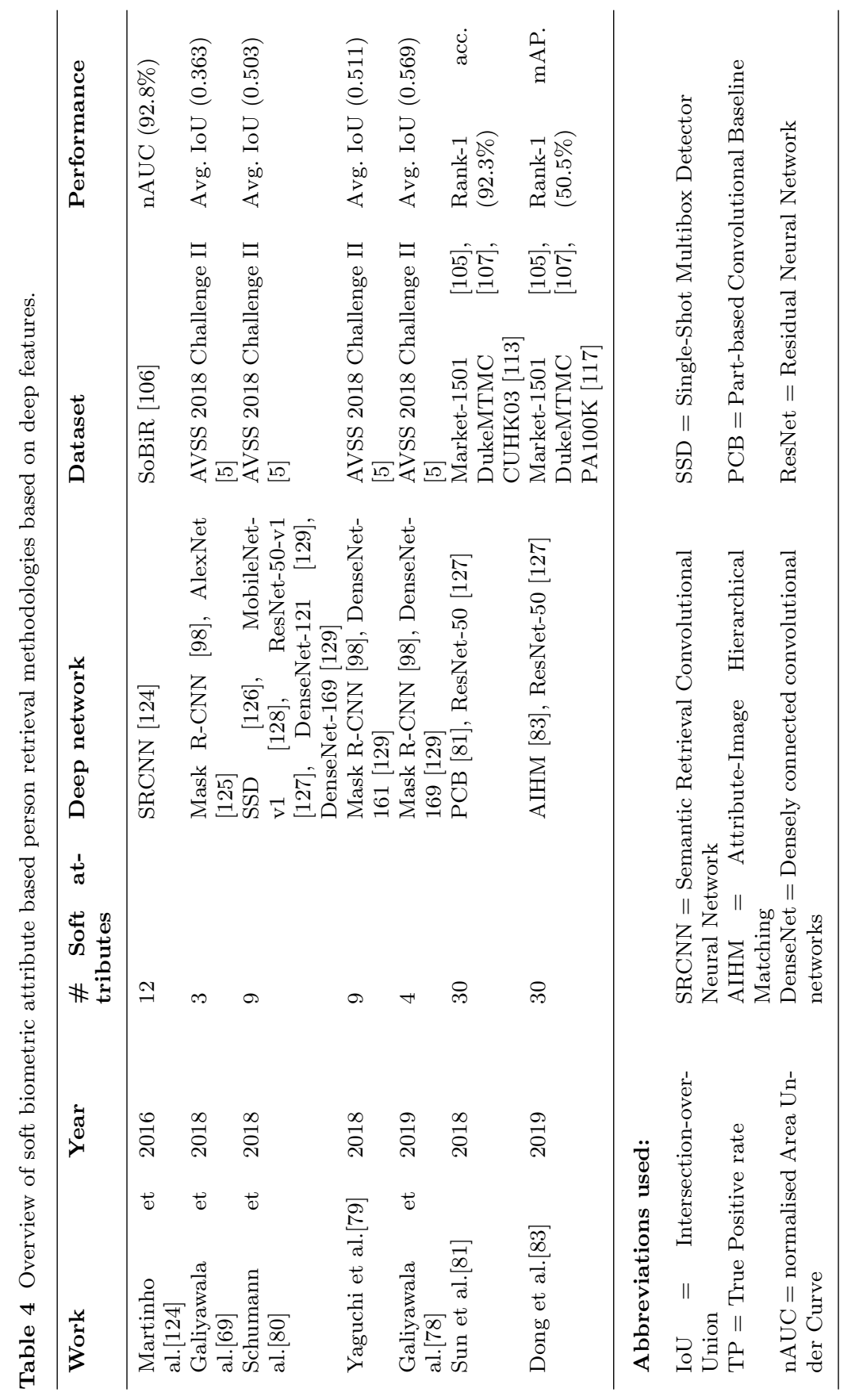



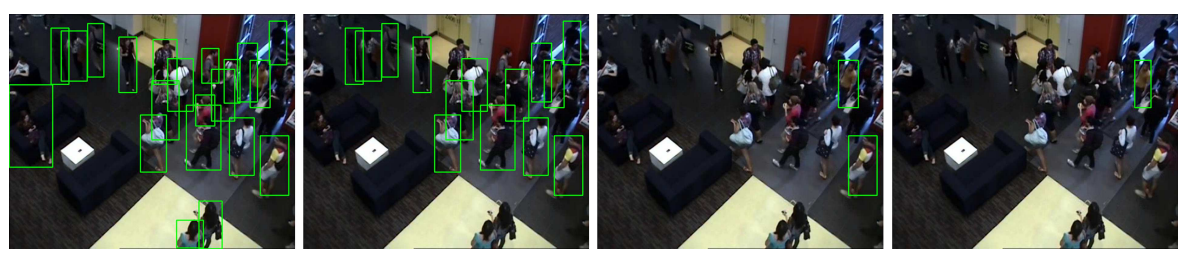

Fig. 16 Sample output frames for the person retrieved using semantic description by approach in [78]: person from Test Sequence 11 and Frame 66 with semantic description height (very short; 140-160 cm), torso type (short sleeve), torso colour-1 (yellow), torso colour-2 (NA) and gender (male). Images from left to right are Mask R-CNN person detection, height filtering, clothing colour filtering and gender filtering.

Yaguchi et al. [79] also use mask R-CNN for person detection and DenseNet161 for attribute classification. Initially, they estimate all the attributes of the detected persons, and then the matching score is calculated using a Hamming loss. The person with the minimum loss is the target. Galiyawala et al. [78] further improve the linear filtering approach of [69] by introducing adaptive torso patch extraction and bounding box regression. Torso patch extraction is undertaken by deciding the torso region according to clothing type attribute. Thus, it removes noisy pixels from the torso patch and provides better colour classification. An IoU based box regression predicts the bounding box in the frame where soft biometric attribute-based retrieval fails. The approaches in [69] and [78] follow a linear filtering approach that filters out the non-matching person according to attributes and leaves the target in the end. The other two methods in [79] and [80] estimate all the detected attributes of a person in parallel. These methods fuse the characteristics in the end to retrieve the target. The linear filtering approach does not need to estimate all the attributes for all detection persons. However, an error in the first filter will propagate to further attribute filtering and reduce retrieval accuracy.

The AVSS 2018 challenge II [5] dataset is evaluated based on two metrics; an average $\mathrm{IoU}$ and percent of frames with an $\mathrm{IoU} \geq 0.4$. State-of-the-art average IoU of 0.569 is achieved by a linear filtering approach [78]. $75.9 \%$ of frames with an $\mathrm{IoU} \geq 0.4$ is achieved by [80]. Some sample qualitative results of person retrieval using the method in [78] are shown in Fig. 16. It offers a surveillance frame from AVSS 2018 challenge II datasets for test sequence 40 and frame 66 with a semantic description, namely, height (very short; 140-160 $\mathrm{cm}$ ), torso type (short sleeve), torso colour-1 (yellow), torso colour-2 (NA) and gender (male). The first image shows the person detection output using Mask R-CNN. Height filter output shows that many persons match the given query. Among these persons, the colour filter output produces a couple of matches and this is further refined by using a gender filter. It is to be noted that all deep features-based approaches [59,78-80] perform better than the handcrafted feature-based baseline approach [76].

Sun et al. [81] apply part level features because they provide fine-grained information for person description. The authors propose a Part-based Convolutional Baseline (PCB) network for part-based feature extraction. Part-level 
Table 5 Comparison of handcrafted and deep feature based methods.

\begin{tabular}{|c|c|c|}
\hline Category & Sub class & Characteristics Advantages/disadvantages \\
\hline \multirow{4}{*}{$\begin{array}{l}\text { Hand } \\
\text { crafted } \\
\text { features }\end{array}$} & Haar $[71]$ & $\begin{array}{l}\text { Face detection works well for near view but it is difficult to } \\
\text { detect for far view and crowded scenarios. }\end{array}$ \\
\hline & $\begin{array}{l}\text { Color his- } \\
\text { togram } \\
{[70,71,73]}\end{array}$ & $\begin{array}{l}\text { Simple to implement and supports the less number of colors } \\
\text { (i.e. primary colors) for classification but difficult if color } \\
\text { classes increases (different shades of color) as it involves } \\
\text { quantization process for histogram calculations. }\end{array}$ \\
\hline & $\begin{array}{l}\text { HOG }[76, \\
110]\end{array}$ & $\begin{array}{l}\text { HOG is good descriptor but its coarseness leads to sub-region } \\
\text { processing in image which increases computational complex- } \\
\text { ity. }\end{array}$ \\
\hline & $\begin{array}{l}\text { Height } \\
{[73-75]}\end{array}$ & $\begin{array}{l}\text { It is calculated based on camera calibration parameters, but } \\
\text { a small error in parameters estimation leads to high error in } \\
\text { height calculations. Moreover, the camera parameter estima- } \\
\text { tion need to done again if camera is moved. }\end{array}$ \\
\hline \multirow[t]{4}{*}{$\begin{array}{l}\text { Deep fea- } \\
\text { tures }\end{array}$} & $\begin{array}{l}\text { AlexNet } \\
{[69]}\end{array}$ & $\begin{array}{l}\text { It is small network and } 61 \mathrm{M} \text { parameters to learn, but the } \\
\text { classification accuracy is low. }\end{array}$ \\
\hline & $\begin{array}{l}\text { MobileNet } \\
{[80]}\end{array}$ & $\begin{array}{l}\text { Its a light weight network and } 4.2 \mathrm{M} \text { parameters to learn, but } \\
\text { it is having little high error rate. }\end{array}$ \\
\hline & $\begin{array}{l}\text { ResNet-50 } \\
{[80,81,83]}\end{array}$ & $\begin{array}{l}\text { It is having advantage of residual block which is having skip } \\
\text { connection from input for better learning ability. Over } 25 \mathrm{M} \\
\text { parameters to learn. }\end{array}$ \\
\hline & $\begin{array}{l}\text { DenseNet } \\
{[78,79,80]}\end{array}$ & $\begin{array}{l}\text { It is having connection from each layer to every other layer } \\
\text { and hence leveraging information from other layers which } \\
\text { learns much better compare to other networks. }\end{array}$ \\
\hline
\end{tabular}

features are learnable by conducting uniform partition on the conv-layer without explicitly partitioning images. The spatial consistency within-part is exploitable to refine the coarse partition provided by PCB. The improvement in the uniform partition is made by the Refined Part Pooling (RPP) network. It achieves $92.3 \%$ of rank-1 accuracy with only the PCB. Further, it improves to $93.8 \%$ of rank-1 accuracy by employing RPP with PCB network on the Market-1501 [105] dataset. The text attribute combinations exist on a large scale in a real scenario. However, a minimal amount of combination with sufficient data is available for training. Except for such combinations, others are never modelled during training. Thus, Dong et al. [83] formulate a textual attribute query-based person search problem as a zero-shot (ZSL) learning problem for the first time. The authors propose Attribute-Image Hierarchical Matching (AIHM), which matches attributes and images at multiple hierarchical levels. The algorithm achieves state-of-the-art rank-1 mAP of $43.3 \%$ on Market-1501 [105], 50.5\% on DukeMTMC [107] and 31.3\% on PA100K [117] datasets. Table 5 shows a comparison of handcrafted and deep feature-based methods with their advantages and disadvantages.

A query-based personal retrieval system usually provides soft biometric keywords as the input query. Improvisation in natural language processing 
now allows automatic extraction of the keywords from sentences. It will move the system towards full automation.

\subsection{Natural language description-based person retrieval}

Cross-modal retrieval-based applications are drawing attention due to the rapid growth of multimodal data like text, image, video, and audio. Features from different modalities like text and image are not directly comparable as they lie entirely in other spaces. Hence, it is a challenging problem due to the sizeable heterogeneous gap between different text and image modalities. One such issue of person retrieval from surveillance video using natural language description is in this section. Table 6 shows an overview of different methodologies for person retrieval using natural language descriptions.

Zhou et al. [89] develop an attention-based algorithm that localises a person in the surveillance frame using attributes and natural language query. The author annotated the cityscapes dataset [132] surveillance frame with attributes and descriptions because the dataset did not have natural language descriptions. Matrix representations of sentence expressions use the Skip-gram model [143]. Attributes and descriptions are by bidirectional Long-Short Term Memory (BLSTM) [130,131] network. Visual features extraction is done using Faster R-CNN [97] and ResNet152 [127] with the algorithm achieving $74.6 \%$ recall@1 i.e., $74.6 \%$ of the highest scoring box is correct. The Cityscape dataset contains only street views, i.e., frontal view camera. Hence, it does not cover various view challenges for surveillance. Also, the description annotated dataset is not available publicly and it limits the usability.

Li et al. [85] first published a large-scale person dataset with natural language description, CUHK-PEDES (publicly available), as discussed in Sect. 2.4. The author proposes a Recurrent Neural Network with a Gated Neural Attention mechanism (GNA-RNN) to establish the baseline on CUHK-PEDES. The network consists of a visual sub-network for visual feature extraction from an image and a language sub-network for textual feature extraction from a description. The visual sub-network consists of VGG-16 [133] as a backbone network and generates 512 graphical units. Each optical unit determines the existence of a specific appearance pattern. RNN with Long Short-Term Memory (LSTM) is useful in language sub-network, which takes words and images as input. It outputs unit level attention that decides which visual units should pay more attention to the word. The word-level gate determines the importance of the word, e.g., the word "white" has more weightage than the word "the". The GNA-RNN network is trained in an end-to-end manner and provides a top-1 accuracy of $19.05 \%$. This approach creates the baseline for further research in cross-modal person retrieval based on natural language descriptions. 


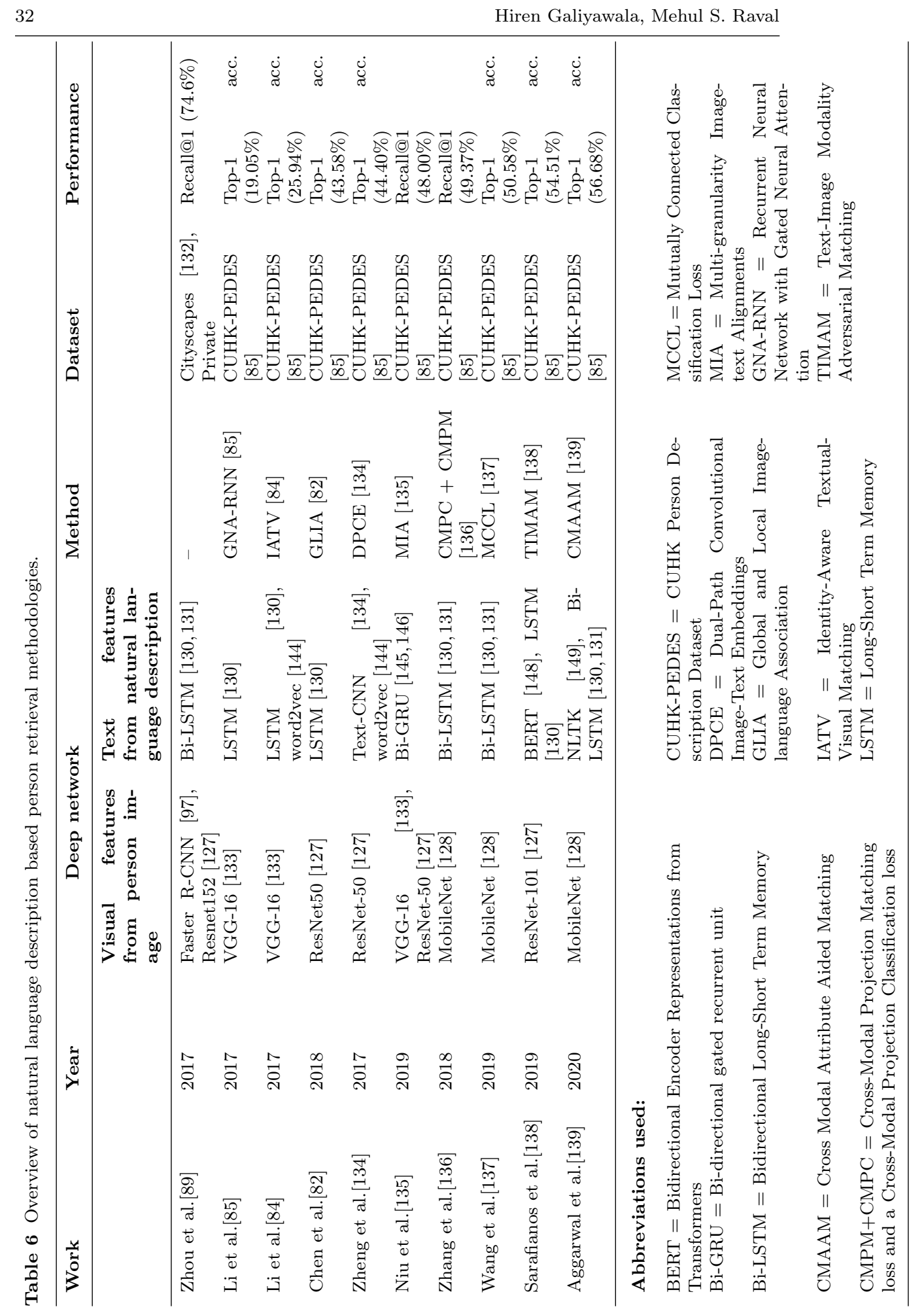


The approaches discussed below are evaluated on this baseline. Most of the large-scale dataset contains identity level annotations considered by $\mathrm{Li}$ et al. [84] to match visual and textual domains. Identity-aware textual-visual matching is done in a two-stage network. Identity-level annotations are effectively utilized by introducing a Cross-Modal Cross-Entropy (CMCE) loss in the stage-1 network. The CMCE loss implicitly maximizes inter-identity feature distances and minimizes intra-identity feature distances. However, the coupling between visual and textual features generated through CMCE loss is loose. Hence, the initial matching of stage- 1 is further refined by stage- 2 CNN-LSTM with an underlying co-attention mechanism that produces the final textual-visual matching confidence. This two-stage framework achieves $25.94 \%$ of top-1 accuracy.

Global and local level image-language association (GLIA) is proposed by Chen et al. [82] to exploit the semantic information available in the description. The GLIA approach gains a significant boost to the top- 1 accuracy from the baselines and achieves $43.58 \%$ top-1 accuracy. Zheng et al. [134] focus on the limitation of ranking loss. The authors do not explicitly consider the feature distribution in a single modality. They overcome it by considering the problem as instance-level retrieval and propose the instance loss. Each image-text query pair is regarded as an instance and observed as a class during training to learn finer granularity. Instead of considering pre-trained models for feature extraction, the method view adopts end-to-end learning from the data itself. Dual-path CNN-CNN i.e. Dual-Path Convolutional Image-Text Embedding (DPCE) architecture is proposed instead of the CNN-RNN approach for image-text matching. DPCE [134] achieves $44.40 \%$ of top-1 rank accuracy.

Multi-granularity Image-text Alignments (MIA) framework [135] adopts a multiple granularities (i.e., global-global, global-local, and local-local alignments) based approach for better similarity evaluations between text and image. The global context of image and description matches global-global granularity. On the other hand, relations between the global context and local components establishes the global-local alignment. Visual human parts fit with noun phrases in the final local-local granularity. The algorithm achieves $48.00 \%$ of recall@1. Zhang et al. [136] focus on learning discriminative features by proposing two loss functions, i.e., cross-modal projection matching (CMPM) loss and a cross-modal projection classification (CMPC) loss. Natural language description is first tokenized into words and processed sequentially using Bi-LSTM. Visual features are extractable from the last pooling layer of MobileNet [128]. The association module embeds visual and textual elements into a shared latent space. $49.37 \%$ of recall@1 is achieved by the algorithm while considering both CMPM and CMPC losses. Similar to work in [84], Wang et al. [137] also utilize identity-level information and propose Mutually Connected Classification Loss (MCCL). They first create a baseline approach before applying MCCL for better feature embedding. This baseline approach uses MobileNet pre-trained on ImageNet [147] for visual features, Bi-LSTM for textual element, and triplet loss function for cross-modal feature embedding. Triplet loss does not fully exploit feature distribution. The MCCL classifica- 
tion weight is shared between both modalities. Only the baseline approach with triplet loss achieves $45.55 \%$ of recall@1 while MCCL achieves $50.58 \%$ of recall@1.

The majority of approaches so far introduce a new loss function for the network to learn better feature representations without the complexity of textual phrases. For example, the word "t-shirt" is an adjective, but it might be useful as a noun in the description. Such limitations go away by introducing a Text-Image Modality Adversarial Matching (TIMAM) framework [138]. Sarafianos et al. [138] propose adversarial representation learning, which helps bring features from different modalities very close. TIMAM attains top-1 accuracy of $54.51 \%$. Aggarwal et al. [139] use attribute classification as an additional task and identity for bridging the gap between different modalities to improve representation learning. The method uses Deep Coral loss [150] to reduce the modality gap. They achieve state-of-the-art top-1 accuracy of $56.61 \%$ on CUHK-PEDES dataset. Thus, most of the work [82,84,85,134-139] shows cross-modal person retrieval on the only publicly available CHUHK-PEDES dataset except [89]. CUHK-PEDES contains only the image gallery of cropped persons, limiting practical usage in real-time scenarios to retrieve the person from the input of full surveillance frames.

\section{Conclusions and open research challenges}

Security of society has been a significant concern over the years, and biometricbased security has gained prominence due to exhaustive research in the field. Biometric security has evolved through the following stages:

- Utilizing hard biometrics [15,16].

- Use of soft biometrics to improve the hard biometric-based systems [6].

- Soft biometric attribute-based person retrieval [7-13].

- Natural language description-based person retrieval [82,84,85,134-139] inherently uses soft biometrics in natural language description.

It has progressed from constrained environment-based fingerprint and face recognition systems to surveillance video-based person retrieval in the wild.

There are mainly two types of person retrieval methods seen in current research: discrete attribute-based (handcrafted feature-based and deep featurebased) methods and natural language description-based methods. Each has advantages and disadvantages. Handcrafted feature-based methods have limited performance due to less discriminative power of hand-engineered features and limited support to more classes for classification. Deep features are much more robust and produce better retrieval results but require a high amount of training data and high computation resources. Discrete attributes are easy to acquire, but do not suit real-time applications where the natural language descriptions are typically used. Most natural language description-based methods (Table 6) are not evaluated on full-frame surveillance videos. Moreover, 
the dataset is also a key element in any system development, including realtime scenarios. Hence, the current research hotspot is to develop an end-to-end person retrieval system from unconstrained surveillance videos.

Given the comprehensive review, some open problems that require further research and analysis are as follows:

1. Diverse dataset development: This review focuses on person retrieval from surveillance videos using soft biometrics, which are in the form of either a discrete attribute or natural language description. Both forms of query are in the form of text to the person retrieval system. The development of various person retrieval methodologies would not have been possible without the continuous evolution of challenging datasets (Table 2) proposed by active researchers in the vision and language field. The datasets cover the following challenges:

- Different resolutions

- Varying illumination conditions

- Occlusion

- Merging foreground with background

- Viewpoint variations

- Crowded scene

- Indoor and outdoor environments

- Time consistency/time duration

- Type of camera or multiple cameras

These challenges (discussed in Sec. 2.1) are not present in all datasets, but they lack one or the other difficulty. For example, CUHK-PEDES is the only large-scale dataset available for cross-modal person retrieval. However, it lacks full surveillance frames and samples of interest merging with background and crowd scenarios.

Issues involved in person detection in regular and crowded scenarios cannot be evaluated due to non-availability of full surveillance frames. AVSS 2018 challenge II is the only dataset that has low-resolution full surveillance frames and covers many challenges. However, it also lacks outdoor scenes and natural language descriptions. Thus, it also cannot be tested against various natural outdoor conditions and limits the development of a robust end-to-end cross-modal person retrieval framework. The more recent PDESTRE dataset provides $4 \mathrm{~K}$ videos but lacks in indoor environment. The majority of the datasets consist of very few samples with occlusion and a mixture of indoor and outdoor environment samples of the same person. Thus, this review of a promising large-scale dataset provides an opportunity to develop a more challenging dataset that can overcome the limitations of an individual dataset.

2. Developing robust person attribute recognition model: As discussed in Sect. 2.3, person attribute recognition is the most critical task for visual attribute extraction. This task uses a cropped person dataset for model preparations. But the attribute model trained on one dataset may not achieve a good performance for other datasets due to lack of diversity. Dif- 


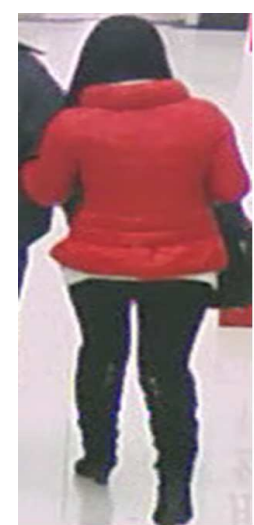

(a)

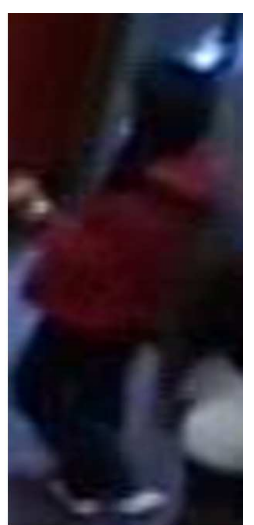

(b)

Fig. 17 Images from two different dataset: (a) RAP [108], (b) AVSS 2018 challenge II [5].

ferences in images from different datasets in terms of colour, view, pose, and illumination conditions are readily observable in Fig. 17. The model training has to be done by merging samples from a different dataset. But annotations to the images from other datasets are not the same. For example, RAP images are annotated with clothing type with 10 classes, namely, $\{$ ubShirt, ub-Sweater, ub-Vest, ub-Tshirt, ub-Cotton, ub-Jacket, ub-SuitUp, ub-Tight, ub-ShortSleeve, ub-Other $\}$. In comparison, AVSS 2018 challenge II images are annotated with clothing type with 3 classes, namely, \{long sleeve, short sleeve, no sleeve $\}$. The solution is that these annotations need to be mapped by annotating one of the datasets with similar annotations to other dataset classes.

Class imbalance is also a problem, e.g., samples for every clothing type may not be of the same number. The number of samples for clothing type in RAP dataset are $\{14652,5260,3209,15640,7814,19209,2057,2830,6549$, $1840\}$ [108]. Such a class imbalance problem can be resolved by weighting each class during the training. Assign a higher weight to a class with fewer samples and a lower weight to a class with more samples.

The total number of training images in the RAP dataset is 67943 , while AVSS has approximately 14000 images. It is observed from Fig. 17 that the RAP dataset images have appropriate illumination conditions and resolutions. However, AVSS dataset images have challenging illumination conditions and lower resolutions. Thus, models trained by merging such datasets will produce good accuracy for clear images like RAP but not for images like the AVSS dataset. Such a problem can be resolved by generating images (for the dataset having fewer images) using domain adaption methodologies as discussed in [160,161].

3. Robust framework development: Different methodologies are reviewed (Table 3, 4, 5) on the dataset mentioned in Table 2. State-of-the-art performance on AVSS 2018 challenge II datasets in terms of average IoU is 0.569 
achieved by the linear filtering approach of [78] and an approach in [80] achieves $75.9 \%$ of frames with an $\mathrm{IoU} \geq 0.4$. The method in [78] has a limitation of error propagation due to the sequential processing of samples and a significantly lower soft biometric amount. Such a process also require to create a separate model for individual attributes, e.g., gender model and colour model. It is not feasible and also a very costly solution to make a different model for every specific attribute. A single model for attribute recognition by multi-attribute learning (as discussed Sec. 2.3) helps resolve such a problem by considering the solutions suggested above to develop a robust person attribute recognition model.

4. Adversarial learning-based approach: Although datasets are available with increasing complexity, e.g., number of persons, images, and attributes, such a cross-modal retrieval problem covers many possible real-scenarios, e.g., the backpack carried by a person in one camera view may not be visible in some other camera views. It is also expensive and tedious to cover many attribute combinations with image annotations in a dataset. Thus, image and text, both modalities together, produce many unseen scenarios (which are not available in the dataset) for person retrieval. It limits the usability and scalability of real-time deployments. Researchers in [156-159] propose adversarial learning-based approaches to mitigate such issues. Yin et al. [156] suggest that attribute-based concept generation learns a discriminative joint space for image and text. Wu et al. [157]propose a deep adversarial data augmentation method with attribute (DADAA), which regularises training by generating unseen scenarios of person images. Unseen attribute combinations are used by a symbiotic adversarial learning (SAL) [159] framework to synthesize features of unseen categories and also to optimise feature embedding by cross-modal alignment in the common embedding space. Hence, data augmentation, unseen attribute combination and attribute-based image generation using adversarial learning for person retrieval are also exciting areas for further research.

5. Backbone network explorations: The majority of the works reviewed in Tables 4, 5 use VGG-16, ResNet, DenseNet, and MobileNet, which are popular and provide state-of-the-art results. Nevertheless, the supremacy of CNN networks like EfficientNets [151] is as yet unexplored. None of the methods utilise EfficientNets, which are smaller and faster than VGG-16, ResNet, DenseNet, and MobileNet. For example, EfficientNet-B0 uses 5.3M parameters which are much less in comparison with ResNet-50 (26M) and DenseNet-169 (14M) while evaluating on ImageNet dataset [147]. Thus, a review of state-of-the-art methodologies and deep network creates a scope to develop a more robust network with better feature representation to improve performance. Hence, person retrieval in surveillance using a textual query is still a challenging and open research problem.

In summary, soft biometrics bridges the gap between human language descriptions and machines to automate the person retrieval process. The application domain ranges from crime investigation, security to missing person 
retrieval. There are multiple soft biometric attributes that can be used for person retrieval, and thus the paper offers a way of selecting an attribute that will help the naive researcher begin research in the domain. The study includes the perspective of challenging datasets, leading edge methods, and deep networks. The paper presents an in-depth review by tracing the development of retrieval in chronological order using handcrafted features, simple attributebased retrieval, and natural language-based retrieval. It guides beginners to gather essential ingredients for their research journey and, at the same time, provides an expert bird's eye view of the domain through a well-conceived summary. The goal of the comprehensive discussion undertaken in this paper about large-scale datasets and various existing problem definitions is to engender motivation to solve problems that exist in this domain.

Acknowledgements The Board of Research in Nuclear Sciences (BRNS), Government of India (36(3)/14/20/2016-BRNS/36020) supports this work. The authors acknowledge the support of NVIDIA Corporation for a donation of the Quadro K5200 GPU used for this research. The authors are thankful to Ahmedabad University, India, for access to resources like GPUs. We would also like to thank the vision and language domain's active researchers for creating publicly available challenging datasets.

\section{References}

1. Bertillon (1889). Instructions for taking descriptions for the identification of criminals and others, by means of anthropometric indications. American Bertillon Prison Bureau.

2. H. T. F. Rhodes(1956). Alphonse Bertillon: Father of scientific detection. AbelardSchuman, New York.

3. https://en.wikipedia.org/wiki/Alphonse_Bertillon. Accessed 28 April 2020.

4. Woodward JD, Orlans NM, Higgins PT (2003). Biometrics. The McGraw-Hill Companies, Inc.

5. Halstead M, Denman S, Fookes C, Tian Y, Nixon MS (2018). Semantic person retrieval in surveillance using soft biometrics: Avss 2018 challenge II. In Proceedings of 15th IEEE International Conference on Advanced Video and Signal Based Surveillance (AVSS), pp. 1-6, Auckland, New Zealand, 2018 Nov 27.

6. Jain AK, Dass SC, Nandakumar K (2004). Soft biometric traits for personal recognition systems. In Proceedings of International Conference on Biometric Authentication (ICBA), pp. 731-738, Springer, Berlin, Heidelberg.

7. Samangooei S, Guo B, Nixon MS (2008). The use of semantic human description as a soft biometric. In Proceedings of 2nd IEEE International Conference on Biometrics: Theory, Applications, and Systems, pp. 1-7, Arlington, USA, 29 Sept.-1 Oct 2008.

8. Reid DA, Nixon MS (2010). Imputing human descriptions in semantic biometrics. In Proceedings of 2nd workshop on Multimedia in forensics, security and intelligence, pp. 25-30, ACM, Firenze, Italy, 29 Oct 2010.

9. Dantcheva A, Velardo C, D'Angelo A, Dugelay JL (2011). Bag of soft biometrics for person identification. Multimedia Tools and Applications, Springer, 51(2):739-777.

10. Reid DA, Samangooei S, Chen C, Nixon MS, Ross A (2013). Soft biometrics for surveillance: an overview. Handbook of statistics, Elsevier, 31:327-352.

11. Nixon MS, Correia PL, Nasrollahi K, Moeslund TB, Hadid A, Tistarelli M (2015). On soft biometrics. Pattern Recognition Letters, Elsevier, 68:218-230.

12. Dantcheva A, Elia P, Ross A (2016). What else does your biometric data reveal? A survey on soft biometrics. IEEE Transactions on Information Forensics and Security, 11(3):441-467.

13. Raval MS (2016). Digital Video Forensics: Description Based Person Identification. CSI Communications, 39(12):9-11. 
14. https://www.bbc.com/news/magazine-22191033. Accessed 28 April 2020.

15. Jain, AK, Ross A, Prabhakar S (2004). An introduction to biometric recognition. IEEE Transactions on circuits and systems for video technology, 14(1):4-20.

16. Jain AK, Flynn P, Ross A (2007). Handbook of biometrics. Springer Science \& Business Media, USA.

17. Tome P, Fierrez J, Vera-Rodriguez R, Nixon MS (2014). Soft biometrics and their application in person recognition at a distance. IEEE Transactions on information forensics and security. 9(3):464-75.

18. Golomb BA, Lawrence DT, Sejnowski TJ (1990). Sexnet: a neural network identifies sex from human faces. In Proceedings of 3rd International Conference on Neural Information Processing Systems (NIPS), pp. 572-577.

19. Gutta S, Wechsler H, Phillips PJ (1998). Gender and ethnic classification of face images. In Proceedings of 3rd IEEE International Conference on Automatic Face and Gesture Recognition, pp. 194-199.

20. Sun Z, Bebis G, Yuan X, Louis SJ (2002). Genetic feature subset selection for gender classification: A comparison study. In Proceedings of 6th IEEE Workshop on Applications of Computer Vision (WACV), pp. 165-170.

21. Moghaddam B, Yang MH (2002). Learning gender with support faces. IEEE Transactions on Pattern Analysis and Machine Intelligence. 24(5):707-11.

22. Jain A, Huang J (2004). Integrating independent components and linear discriminant analysis for gender classification. In Proceedings of 6th IEEE International Conference on Automatic Face and Gesture Recognition, pp. 159-163, 2004 May 19.

23. Sun N, Zheng W, Sun C, Zou C, Zhao L (2006). Gender classification based on boosting local binary pattern. In International Symposium on Neural Networks, pp. 194-201, 2006 May 28, Springer, Berlin, Heidelberg.

24. Kim HC, Kim D, Ghahramani Z, Bang SY (2006). Appearance-based gender classification with Gaussian processes. Pattern Recognition Letters, Elsevier, 27(6):618-626.

25. Bekios-Calfa J, Buenaposada JM, Baumela L (2010). Revisiting linear discriminant techniques in gender recognition. IEEE Transactions on Pattern Analysis and Machine Intelligence, 33(4):858-864.

26. Jia S, Cristianini N (2015). Learning to classify gender from four million images. Pattern recognition letters, Elsevier, 58:35-41.

27. Omidiora EO, Ojo O, Yekini NA, Tubi TA (2012). Analysis, design and implementation of human fingerprint patterns system. Towards age \& gender determination, ridge thickness to valley thickness ratio (rtvtr) \& ridge count on gender detection. International Journal of Advanced Research in Artificial Intelligence, 1(2):5763.

28. Gupta S, Rao AP (2014). Fingerprint based gender classification using discrete wavelet transform \& artificial neural network. International Journal of Computer Science and mobile computing, 3(4):1289-1296.

29. Badawi AM, Mahfouz M, Tadross R, Jantz R (2006). Fingerprint-based gender classification. In Proceedings of International Conference on Image Processing, Computer Vision and Pattern Recognition, pp. 4146.

30. Tom RJ, Arulkumaran T, Scholar ME (2013). Fingerprint based gender classification using 2D discrete wavelet transforms and principal component analysis. International Journal of Engineering Trends and Technology, 4(2):199-203.

31. Marasco E, Lugini L, Cukic B (2014). Exploiting quality and texture features to estimate age and gender from fingerprints. In Biometric and Surveillance Technology for Human and Activity Identification XI, (Vol. 9075, p. 90750F). International Society for Optics and Photonics.

32. Rattani A, Chen C, Ross A (2014). Evaluation of texture descriptors for automated gender estimation from fingerprints. In European Conference on Computer Vision,pp. 764-777, Springer, Cham

33. Thomas V, Chawla NV, Bowyer KW, Flynn PJ (2007). Learning to predict gender from iris images. In 1st IEEE International Conference on Biometrics: Theory, Applications, and Systems, pp. 1-5.

34. Lagree S, Bowyer KW (2011). Predicting ethnicity and gender from iris texture. In IEEE International Conference on Technologies for Homeland Security (HST), pp. 440-445.

35. Cao L, Dikmen M, Fu Y, Huang TS (2008). Gender recognition from body. In Proceedings of the 16th ACM international conference on Multimedia, pp. 725-728. 
36. Chen L, Wang Y, Wang Y (2009). Gender classification based on fusion of weighted multi-view gait component distance. In IEEE Chinese Conference on Pattern Recognition, pp. $1-5$.

37. Li X, Maybank SJ, Yan S, Tao D, Xu D (2008). Gait components and their application to gender recognition. IEEE Transactions on Systems, Man, and Cybernetics, Part C (Applications and Reviews), 38(2):145-155.

38. Yu S, Tan T, Huang K, Jia K, Wu X (2009). A study on gait-based gender classification. IEEE Transactions on image processing, 18(8):1905-1910.

39. Yoo JH, Hwang D, Nixon MS (2005). Gender classification in human gait using support vector machine. In International Conference on Advanced Concepts for Intelligent Vision Systems, pp. 138-145). Springer, Berlin, Heidelberg.

40. Shan C, Gong S, McOwan PW (2008). Fusing gait and face cues for human gender recognition. Neurocomputing, 71(10-12):1931-1938.

41. Kanchan T, Krishan K (2011). Anthropometry of hand in sex determination of dismembered remains-A review of literature. Journal of Forensic and Legal Medicine., 18(1):14-17.

42. Scheuer JL, Elkington NM (1993). Sex determination from metacarpals and the first proximal phalanx. Journal of Forensic Science, 38(4):769-778.

43. Falsetti AB (1995). Sex assessment from metacarpals of the human hand. Journal of Forensic Science, 40(5):774-776.

44. Lazenby RA (1994). Identification of sex from metacarpals: effect of side asymmetry. Journal of Forensic Science, 39(5):1188-1194

45. Krishan K, Kanchan T, Sharma A (2011). Sex determination from hand and foot dimensions in a North Indian population. Journal of forensic sciences, 56(2):453-459.

46. Amayeh G, Bebis G, Nicolescu M (2008). Gender classification from hand shape. In IEEE Computer Society Conference on Computer Vision and Pattern Recognition Workshops, pp. 1-7.

47. Wu K, Childers DG (1991). Gender recognition from speech. Part I: Coarse analysis. The journal of the Acoustical society of America, 90(4):1828-1840.

48. Pronobis M, Magimai-Doss M (2009). Analysis of F0 and cepstral features for robust automatic gender recognition. IDIAP Technical Report.

49. Sorokin VN, Makarov IS (2008). Gender recognition from vocal source. Acoustical Physics, 54(4):571-578.

50. Childers DG, Wu K (1991). Gender recognition from speech. Part II: Fine analysis. The Journal of the Acoustical society of America, 90(4):1841-1856

51. Walawalkar L, Yeasin M, Narasimhamurthy AM, Sharma R (2002). Support vector learning for gender classification using audio and visual cues: A comparison. In International Workshop on Support Vector Machines, pp. 144-159, Springer, Berlin, Heidelberg.

52. Ding L, Martinez AM (2008). Precise detailed detection of faces and facial features. In IEEE Conference on Computer Vision and Pattern Recognition (CVPR), pp. 1-7.

53. Nixon M (1985). Eye spacing measurement for facial recognition. In Applications of Digital Image Processing VIII, 575:279-285, International Society for Optics and Photonics.

54. Ramanathan V, Wechsler H (2010). Robust human authentication using appearance and holistic anthropometric features. Pattern Recognition Letters, 31(15):24252435.

55. Ghalleb AE, Sghaier S, Amara NE (2013). Face recognition improvement using soft biometrics. In 10th International Multi-Conferences on Systems, Signals \& Devices 2013 (SSD13), pp. 1-6.

56. BenAbdelkader C, Davis L (2006). Estimation of anthropomeasures from a single calibrated camera. In 7th International Conference on Automatic Face and Gesture Recognition (FGR06), pp. 499-504)

57. Madden CS, Piccardi M (2005). Height measurement as a session-based biometric for people matching across disjoint camera views. In Image and Vision Computing Conference, Wickliffe Ltd.

58. Bobick AF, Johnson AY (2001). Gait recognition using static, activity-specific parameters. In Proceedings of IEEE Computer Society Conference on Computer Vision and Pattern Recognition (CVPR), 1:I-I).

59. BenAbdelkader C, Cutler R, Davis L (2002). View-invariant estimation of height and stride for gait recognition. In International Workshop on Biometric Authentication, pp. 155-167, Springer, Berlin, Heidelberg. 
60. Li SZ, Schouten B, Tistarelli M (2009). Handbook of Remote Biometrics for Surveillance and Security, pp. 321, Springer-Verlag, New York, USA.

61. Reid DA, Nixon MS, Stevenage SV (2013). Soft biometrics; human identification using comparative descriptions. IEEE Transactions on pattern analysis and machine intelligence,36(6):1216-1228.

62. Tome P, Fierrez J, Vera-Rodriguez R, Nixon MS (2014). Soft biometrics and their application in person recognition at a distance. IEEE Transactions on information forensics and security, 9(3):464-475.

63. Jain AK, Nandakumar K, Lu X, Park U (2004). Integrating faces, fingerprints, and soft biometric traits for user recognition. In International Workshop on Biometric Authentication, pp. 259-269, Springer, Berlin, Heidelberg.

64. Jain AK, Dass SC, Nandakumar K. Can soft biometric traits assist user recognition? (2004). In Biometric technology for human identification, 5404:561-572. International Society for Optics and Photonics.

65. Park U, Jain AK (2010). Face matching and retrieval using soft biometrics. IEEE Transactions on Information Forensics and Security, 5(3):406-415.

66. Jain AK, Park U (2009). Facial marks: Soft biometric for face recognition. In 16th IEEE International Conference on Image Processing (ICIP), pp. 37-40.

67. Lee JE, Jain AK, Jin R. Scars, marks and tattoos (SMT): Soft biometric for suspect and victim identification (2008). In IEEE Biometrics symposium pp. 1-8.

68. Galiyawala HJ, Raval MS, Laddha A (2020). Person retrieval in surveillance videos using deep soft biometrics. In Deep Biometrics, pp. 191-214, Springer, Cham.

69. Galiyawala H, Shah K, Gajjar V, Raval MS (2018). Person retrieval in surveillance video using height, color and gender. In 15th IEEE International Conference on Advanced Video and Signal Based Surveillance (AVSS), pp. 1-6.

70. Shah P, Raval MS, Pandya S, Chaudhary S, Laddha A, Galiyawala H (2017). Description based person identification: use of clothes color and type. In National Conference on Computer Vision, Pattern Recognition, Image Processing, and Graphics (NCVPRIPG) pp. 457-469, Springer, Singapore.

71. Vaquero DA, Feris RS, Tran D, Brown L, Hampapur A, Turk M (2009). Attribute-based people search in surveillance environments. In IEEE workshop on applications of computer vision (WACV), pp. 1-8.

72. Samangooei S, Guo B, Nixon MS (2008). The use of semantic human description as a soft biometric. In 2nd IEEE International Conference on Biometrics: Theory, Applications and Systems, pp. 1-7.

73. Denman S, Fookes C, Bialkowski A, Sridharan S (2009). Soft-biometrics: unconstrained authentication in a surveillance environment. In IEEE Digital Image Computing: Techniques and Applications, pp. 196-203.

74. Denman S, Halstead M, Bialkowski A, Fookes C, Sridharan S (2012). Can you describe him for me? a technique for semantic person search in video. In IEEE International Conference on Digital Image Computing Techniques and Applications (DICTA), pp. 1-8.

75. Halstead M, Denman S, Sridharan S, Fookes C (2014). Locating people in video from semantic descriptions: A new database and approach. In 22nd IEEE International Conference on Pattern Recognition, pp. 4501-4506.

76. Denman S, Halstead M, Fookes C, Sridharan S (2015). Searching for people using semantic soft biometric descriptions. Pattern Recognition Letters, Elsevier, 68:306-15.

77. Doretto G, Sebastian T, Tu P, Rittscher J (2011). Appearance-based person reidentification in camera networks: problem overview and current approaches. Journal of Ambient Intelligence and Humanized Computing, 2(2):127-151.

78. Galiyawala H, Raval MS, Dave S (2019). Visual appearance based person retrieval in unconstrained environment videos. Image and Vision Computing, 92:103816.

79. Yaguchi T, Nixon MS (2018). Transfer learning based approach for semantic person retrieval. In 15th IEEE International Conference on Advanced Video and Signal Based Surveillance (AVSS), pp. 1-6.

80. Schumann A, Specker A, Beyerer J (2018), Attribute-based person retrieval and search in video sequences. In 15th IEEE International Conference on Advanced Video and Signal Based Surveillance (AVSS), pp. 1-6. 
81. Sun Y, Zheng L, Yang Y, Tian Q, Wang S (2018). Beyond part models: Person retrieval with refined part pooling (and a strong convolutional baseline). In Proceedings of the European Conference on Computer Vision (ECCV), pp. 480-496.

82. Chen D, Li H, Liu X, Shen Y, Shao J, Yuan Z, Wang X (2018). Improving deep visual representation for person re-identification by global and local image-language association. In Proceedings of the European Conference on Computer Vision (ECCV), pp. 54-70.

83. Dong Q, Gong S, Zhu X (2019). Person search by text attribute query as zero-shot learning. In Proceedings of the IEEE International Conference on Computer Vision (ICCV), pp. 3652-3661

84. Li S, Xiao T, Li H, Yang W, Wang X (2017). Identity-aware textual-visual matching with latent co-attention. In Proceedings of the IEEE International Conference on Computer Vision (ICCV), pp. 1890-1899.

85. Li S, Xiao T, Li H, Zhou B, Yue D, Wang X (2017). Person search with natural language description. In Proceedings of the IEEE Conference on Computer Vision and Pattern Recognition (CVPR), pp. 1970-1979.

86. Lin Y, Zheng L, Zheng Z, Wu Y, Hu Z, Yan C, Yang Y (2019). Improving person re-identification by attribute and identity learning. Pattern Recognition, 95:151-161.

87. Liu H, Feng J, Jie Z, Jayashree K, Zhao B, Qi M, Jiang J, Yan S (2017). Neural person search machines. In Proceedings of the IEEE International Conference on Computer Vision (ICCV), pp. 493-501.

88. Yamaguchi M, Saito K, Ushiku Y, Harada T (2017). Spatio-temporal person retrieval via natural language queries. In Proceedings of the IEEE International Conference on Computer Vision (ICCV), pp. 1453-1462.

89. Zhou T, Chen M, Yu J, Terzopoulos D (2017). Attention-based natural language person retrieval. In Proceedings of the IEEE Conference on Computer Vision and Pattern Recognition Workshops (CVPR), pp. 27-34.

90. Chang TH, Gong S (2001). Tracking multiple people with a multi-camera system. In Proceedings IEEE Workshop on Multi-Object Tracking, pp. 19-26.

91. Denman SP, Chandran V, Sridharan S (2017). Robust real time multi-layer foreground segmentation. In Proceedings of International Association for Pattern Recognition (IAPR) Conference on Machine Vision Applications, pp. 496-499.

92. Huang $\mathrm{CH}, \mathrm{Wu}$ YT, Shih MY (2009). Unsupervised pedestrian re-identification for loitering detection. In 3rd Pacific-Rim Symposium on Image and Video Technology, pp. 771-783, Springer, Berlin, Heidelberg.

93. Bak S, Corvee E, Bremond F, Thonnat M (2010). Person re-identification using spatial covariance regions of human body parts. In 7th IEEE International Conference on Advanced Video and Signal Based Surveillance, pp. 435-440.

94. Redmon J, Divvala S, Girshick R, Farhadi A (2016). You only look once: Unified, realtime object detection. In Proceedings of the IEEE conference on computer vision and pattern recognition (CVPR), pp. 779-788.

95. Girshick R, Donahue J, Darrell T, Malik J (2014). Rich feature hierarchies for accurate object detection and semantic segmentation. In Proceedings of the IEEE conference on computer vision and pattern recognition (CVPR), pp. 580-587.

96. Girshick R (2015). Fast r-cnn. In Proceedings of the IEEE International conference on computer vision (ICCV), pp. 1440-1448.

97. Ren S, He K, Girshick R, Sun J (2015). Faster r-cnn: Towards real-time object detection with region proposal networks. In Advances in neural information processing systems (NIPS), pp. 91-99.

98. K. He, G. Gkioxari, P. Dollar, R. Girshick (2017). Mask R-CNN. In IEEE International Conference on Computer Vision (ICCV), pp. 29802988.

99. Gray D, Brennan S, Tao H(2007). Evaluating appearance models for recognition, reacquisition, and tracking. In Proceedings of IEEE International workshop on performance evaluation for tracking and surveillance (PETS), 3(5):4147, Citeseer.

100. Hirzer M, Beleznai C, Roth PM, Bischof H (2011). Person re-identification by descriptive and discriminative classification. In Scandinavian conference on Image analysis, pp. 91-102, Springer, Berlin, Heidelberg.

101. Loy CC, Xiang T, Gong S (2010). Time-delayed correlation analysis for multi-camera activity understanding. International Journal of Computer Vision, 90(1):106-129. 
102. Layne R, Hospedales TM, Gong S (2012). Person re-identification by attributes. In British Machine Vision Conference (BMVC), British Machine Vision Association, 2(3):8.R.

103. Layne R, Hospedales TM, Gong S (2014). Attributes-based re-identification. In Person Re-identification, pp. 93117, Springer, London.

104. Deng Y, Luo P, Loy CC, Tang X (2014). Pedestrian attribute recognition at far distance. In Proceedings of the 22nd ACM international conference on Multimedia, pp. 789792.

105. Zheng L, Shen L, Tian L, Wang S, Wang J, Tian Q (2015). Scalable person reidentification: A benchmark. In Proceedings of the IEEE international conference on computer vision (ICCV), pp. 1116-1124.

106. Martinho-Corbishley D, Nixon MS, Carter JN (2016). Soft biometric retrieval to describe and identify surveillance images. In IEEE International Conference on Identity, Security and Behavior Analysis (ISBA), pp. 1-6.

107. Ristani E, Solera F, Zou R, Cucchiara R, Tomasi C (2016). Performance measures and a data set for multi-target, multi-camera tracking. In European Conference on Computer Vision (ECCV), pp. 17-35, Springer, Cham.

108. Li D, Zhang Z, Chen X, Huang K(2018). A richly annotated pedestrian dataset for person retrieval in real surveillance scenarios. IEEE transactions on image processing, 28(4):1575-1590.

109. Li D, Zhang Z, Shan C, Wang L, Tan T (2019). A Comprehensive Study on Large-Scale Person Retrieval in Real Surveillance Scenarios. In 16th IEEE International Conference on Advanced Video and Signal Based Surveillance (AVSS),pp. 1-8.

110. Zhu J, Liao S, Lei Z, Yi D, Li S (2013). Pedestrian attribute classification in surveillance: Database and evaluation. In Proceedings of the IEEE International Conference on Computer Vision (ICCV) Workshops, pp. 331-338.

111. Baltieri D, Vezzani R, Cucchiara, R (2011). 3dpes: 3d people dataset for surveillance and forensics. In Proceedings of the joint ACM workshop on Human gesture and behavior understanding, pp. 59-64

112. Cheng DS, Cristani M, Stoppa M, Bazzani L, Murino V (2011). Custom Pictorial Structures for Re-identification. In British Machine Vision Conference (BMVC), 1(2):p. 6.

113. Li W., Zhao R., Xiao T, Wang X (2014). Deepreid: Deep filter pairing neural network for person re-identification. In Proceedings of the IEEE Conference on Computer Vision and Pattern Recognition (CVPR), pp. 152-159.

114. Wang T, Gong S, Zhu X, Wang S (2014). Person re-identification by video ranking. In European conference on computer vision (ECCV), pp. 688-703, Springer, Cham.

115. Baltieri D, Vezzani R, Cucchiara R (2011). Sarc3d: a new 3d body model for people tracking and re-identification. In International conference on image analysis and processing, pp. 197-206, Springer, Berlin, Heidelberg.

116. Benfold B, Reid I (2011). Stable multi-target tracking in real-time surveillance video. In In Proceedings of the IEEE conference on computer vision and pattern recognition (CVPR), pp. 3457-3464.

117. Liu X, Zhao H, Tian M, Sheng L, Shao J, Yi S, Yan J, Wang X (2017). Hydraplus-net: Attentive deep features for pedestrian analysis. In Proceedings of the IEEE international conference on computer vision (ICCV), pp. 350-359.

118. Zheng L, Zhang H, Sun S, Chandraker M, Yang Y, Tian Q (2017). Person reidentification in the wild. In Proceedings of the IEEE Conference on Computer Vision and Pattern Recognition (CVPR), pp. 1367-1376.

119. Anguelov D, Lee KC, Gokturk SB, Sumengen B (2007). Contextual identity recognition in personal photo albums. In IEEE Conference on Computer Vision and Pattern Recognition (CVPR), pp. 1-7).

120. Tsai R (1987). A versatile camera calibration technique for high-accuracy 3D machine vision metrology using off-the-shelf TV cameras and lenses. IEEE Journal on Robotics and Automation, 3(4):323-344

121. Thirde D, Li L, Ferryman J (2006). An overview of the pets 2006 dataset. In International Workshop on Performance Evaluation of Tracking and Surveillance, pp. 47-50.

122. Kelly KL, Judd DB (1976). Color: universal language and dictionary of names. US Department of Commerce, National Bureau of Standards. 
123. Sharma G, Wu W, Dalal EN (2005). The CIEDE2000 colordifference formula: Implementation notes, supplementary test data, and mathematical observations. Color Research \& Application: Endorsed by InterSociety Color Council, The Colour Group (Great Britain), Canadian Society for Color, Color Science Association of Japan, Dutch Society for the Study of Color, The Swedish Colour Centre Foundation, Colour Society of Australia, Centre Franais de la Couleur, 30(1):21-30.

124. Martinho-Corbishley D, Nixon MS, Carter JN (2016). Retrieving relative soft biometrics for semantic identification. In 23rd IEEE International Conference on Pattern Recognition (ICPR), pp. 3067-3072).

125. Krizhevsky A, Sutskever I, Hinton GE (2012). Imagenet classification with deep convolutional neural networks. In Advances in neural information processing systems (NIPS), pp. 1097-1105.

126. Liu W, Anguelov D, Erhan D, Szegedy C, Reed S, Fu CY, Berg AC (2016). SSD: Single shot multibox detector. In European conference on computer vision (ECCV), pp. 21-37, Springer, Cham.

127. He K, Zhang X, Ren S, Sun J (2016). Deep residual learning for image recognition. In Proceedings of the IEEE conference on computer vision and pattern recognition (CVPR), pp. 770-778

128. Howard AG, Zhu M, Chen B, Kalenichenko D, Wang W, Weyand T, Andreetto M, Adam H (2017). Mobilenets: Efficient convolutional neural networks for mobile vision applications. arXiv preprint arXiv:1704.04861.

129. Huang G, Liu Z, Van Der Maaten L, Weinberger KQ (2017). Densely connected convolutional networks. In Proceedings of the IEEE conference on computer vision and pattern recognition (CVPR), pp. 4700-4708.

130. Hochreiter S, Schmidhuber J (1997). Long short-term memory. Neural computation, $9(8): 1735-1780$.

131. Schuster M, Paliwal KK (1997). Bidirectional recurrent neural networks. IEEE transactions on Signal Processing, 45(11):2673-2681.

132. Cordts M, Omran M, Ramos S, Rehfeld T, Enzweiler M, Benenson R, Franke U, Roth S, Schiele B (2016). The cityscapes dataset for semantic urban scene understanding. In Proceedings of the IEEE conference on computer vision and pattern recognition, pp. 3213-3223.

133. Simonyan K, Zisserman A (2014). Very deep convolutional networks for large-scale image recognition. arXiv preprint arXiv:1409.1556.

134. [134]. Zheng Z, Zheng L, Garrett M, Yang Y, Shen YD (2017). Dual-path convolutional image-text embedding with instance loss. arXiv preprint arXiv:1711.05535.

135. Niu K, Huang Y, Ouyang W, Wang L (2020). Improving description-based person reidentification by multi-granularity image-text alignments. IEEE Transactions on Image Processing, 29:5542-5556.

136. Zhang Y, Lu H (2018). Deep cross-modal projection learning for image-text matching. In Proceedings of the European Conference on Computer Vision (ECCV), pp. 686-701.

137. Wang Y, Bo C, Wang D, Wang S, Qi Y, Lu H (2019). Language Person Search with Mutually Connected Classification Loss. In IEEE International Conference on Acoustics, Speech and Signal Processing (ICASSP), pp. 2057-2061).

138. Sarafianos N, Xu X, Kakadiaris IA (2019). Adversarial Representation Learning for Text-to-Image Matching. In Proceedings of the IEEE International Conference on Computer Vision (ICCV), pp. 5814-5824.

139. Aggarwal S, RADHAKRISHNAN VB, Chakraborty A (2020). Text-based Person Search via Attribute-aided Matching. In IEEE Winter Conference on Applications of Computer Vision (WACV), pp. 2617-2625.

140. Wang K, Yin Q, Wang W, Wu S, Wang L (2016). A comprehensive survey on crossmodal retrieval. arXiv preprint arXiv:1607.06215.

141. Zhen L, Hu P, Wang X, Peng D (2019). Deep supervised cross-modal retrieval. In Proceedings of the IEEE Conference on Computer Vision and Pattern Recognition (CVPR) pp. 10394-10403.

142. Hu P, Peng D, Wang X, Xiang Y (2019). Multimodal adversarial network for crossmodal retrieval. Knowledge-Based Systems, 180:38-50. 
143. Mikolov T, Sutskever I, Chen K, Corrado GS, Dean J (2013). Distributed representations of words and phrases and their compositionality. In Advances in neural information processing systems (NIPS) pp. 3111-3119.

144. Mikolov T, Chen K, Corrado G, Dean J (2013). Efficient estimation of word representations in vector space. arXiv preprint arXiv:1301.3781.

145. Cho K, van Merrinboer B, Bahdanau D, Bengio Y (2014). On the Properties of Neural Machine Translation: EncoderDecoder Approaches. In Proceedings of SSST-8, Eighth Workshop on Syntax, Semantics and Structure in Statistical Translation, pp. 103-111.

146. Chung J, Gulcehre C, Cho K, Bengio Y (2014). Empirical evaluation of gated recurrent neural networks on sequence modeling. In NIPS 2014 Workshop on Deep Learning.

147. Deng J, Dong W, Socher R, Li LJ, Li K, Fei-Fei L (2009). Imagenet: A large-scale hierarchical image database. In IEEE Conference on Computer Vision and Pattern Recognition (CVPR), pp. 248-255.

148. Devlin J, Chang MW, Lee K, Toutanova K (2018). Bert: Pre-training of deep bidirectional transformers for language understanding. arXiv preprint arXiv:1810.04805.

149. Loper E, Bird S (2002). Nltk: The natural language toolkit. In ACL-02 Workshop on Effective Tools and Methodologies for Teaching Natural Language Processing and Computational Linguistics, ETMTNLP 02. Association for Computational Linguistics.

150. Sun B, Saenko K (2016). Deep coral: Correlation alignment for deep domain adaptation. In European conference on computer vision (ECCV), pp. 443-450. Springer, Cham.

151. Tan M, Le Q (2019). EfficientNet: Rethinking Model Scaling for Convolutional Neural Networks. In International Conference on Machine Learning (ICML), pp. 6105-6114.

152. Li D, Chen X, Huang K (2015). Multi-attribute learning for pedestrian attribute recognition in surveillance scenarios. In IEEE IAPR Asian Conference on Pattern Recognition (ACPR), pp. 111-115.

153. Sudowe P, Spitzer H, Leibe B (2015). Person attribute recognition with a jointly-trained holistic cnn model. In Proceedings of the IEEE International Conference on Computer Vision (ICCV) Workshops, pp. 87-95.

154. Zhu J, Liao S, Yi D, Lei Z, Li SZ (2015). Multi-label cnn based pedestrian attribute learning for soft biometrics. In IEEE International Conference on Biometrics (ICB), pp. 535-540.

155. Kumar SV, Yaghoubi E, Das A, Harish BS, Proena H (2020). The P-DESTRE: A Fully Annotated Dataset for Pedestrian Detection, Tracking, Re-Identification and Search from Aerial Devices. arXiv preprint arXiv:2004.02782.

156. Yin Z, Zheng WS, Wu A, Yu HX, Wan H, Guo X, Huang F, Lai J (2018). Adversarial attribute-image person re-identification. In Proceedings of the 27 th International Joint Conference on Artificial Intelligence, pp. 1100-1106.

157. Wu Q, Dai P, Chen P, Huang Y (2019). Deep adversarial data augmentation with attribute guided for person re-identification. Signal, Image and Video Processing, 5:1-8, Springer.

158. Zha ZJ, Liu J, Chen D, Wu F (2020). Adversarial attribute-text embedding for person search with natural language query. IEEE Transactions on Multimedia, 22(7): 1836-1846.

159. Cao YT, Wang J, Tao D (2020). Symbiotic Adversarial Learning for Attribute-based Person Search. arXiv preprint arXiv:2007.09609.

160. Wei L, Zhang S, Gao W, Tian Q (2018). Person transfer gan to bridge domain gap for person re-identification. In Proceedings of the IEEE Conference on Computer Vision and Pattern Recognition (CVPR), pp. 79-88.

161. Khatun A, Denman S, Sridharan S, Fookes C (2020). End-to-End Domain Adaptive Attention Network for Cross-Domain Person Re-Identification. arXiv preprint arXiv:2005.03222. 